\title{
QUADRATIC SYSTEMS WITH A RATIONAL FIRST INTEGRAL OF DEGREE THREE: A COMPLETE CLASSIFICATION IN THE COEFFICIENT SPACE $\mathbb{R}^{12}$
}

\author{
JOAN C. ARTÉS ${ }^{1}$, JAUME LLIBRE ${ }^{1}$ AND NICOLAE VULPE ${ }^{2}$
}

\begin{abstract}
A quadratic polynomial differential system can be identified with a single point of $\mathbb{R}^{12}$ through its coefficients. The phase portrait of the quadratic systems having a rational first integral of degree 3 have been studied using normal forms. Here using the algebraic invariant theory, we characterize all the non-degenerate quadratic polynomial differential systems of $\mathbb{R}^{12}$ having a rational first integral of degree 3 . We show that there are only 31 different topological phase portraits in the Poincare disc associated to this family of quadratic systems up to a reversal of the sense of their orbits, and we provide representatives of every class modulo an affine change of variables and a rescaling of the time variable. Moreover, each one of these 31 representatives is determined by a set of algebraic invariant conditions and we provide for it a first integral.
\end{abstract}

\section{INTRODUCTION}

Let $\mathbb{R}[x, y]$ be the ring of the polynomials in the variables $x$ and $y$ with coefficients in $\mathbb{R}$. We consider a system of polynomial differential equations or simply a polynomial differential system in $\mathbb{R}^{2}$ defined by

$$
\begin{aligned}
& \dot{x}=P(x, y), \\
& \dot{y}=Q(x, y),
\end{aligned}
$$

where $P, Q \in \mathbb{R}[x, y]$. We say that the maximum of the degrees of the polynomials $P$ and $Q$ is the degree of system (1). A quadratic polynomial differential system or simply a quadratic system is a polynomial differential system of degree 2 . We say that the quadratic system (1) is non-degenerate if the polynomials $P$ and $Q$ are relatively prime or coprime.

Let $U$ be an open and dense subset of $\mathbb{R}^{2}$, we say that a nonconstant function $\mathcal{H}: U \rightarrow \mathbb{R}$ is a first integral of system (1) on $U$ if $\mathcal{H}(x(t), y(t))$ is constant for all of the values of $t$ for which $(x(t), y(t))$ is a solution of system (1). Obviously $\mathcal{H}$ is a first integral of system (1) if and only if

$$
P(x, y) \frac{\partial \mathcal{H}}{\partial x}(x, y)+Q(x, y) \frac{\partial \mathcal{H}}{\partial y}(x, y)=0,
$$

for all $(x, y) \in U$. When a polynomial differential system (1) has a first integral we say that the system is integrable.

On the other hand given $f \in \mathbb{R}[x, y]$ we say that the curve $f(x, y)=0$ is an algebraic invariant curve of system (1) if there exists $K \in \mathbb{R}[x, y]$ such that

$$
P \frac{\partial f}{\partial x}+Q \frac{\partial f}{\partial y}=K f
$$

The name of invariant for such an algebraic curve $f(x, y)=0$ is due to the fact that if a trajectory has a point on $f(x, y)=0$, then the whole trajectory is contained in $f(x, y)=0$.

The search of first integrals is a classic tool in order to describe the phase portraits of a planar differential system. As usual the phase portrait of a differential system is the decomposition of the domain of definition of the system as union of all its orbits or trajectories.

1991 Mathematics Subject Classification. Primary 34C05, 34A34.

Key words and phrases. quadratic vector fields, integrability, rational first integral, phase portraits. 
If the first integral $\mathcal{H}$ is a rational function, then we say that $\mathcal{H}$ is a rational first integral. For a rational first integral we always assume that the polynomial of its numerator is coprime with the polynomial of its denominator. If the maximum between the degrees of the polynomials of the numerator and denominator of a rational first integral is $m$, then we say that the rational first integral $\mathcal{H}$ has degree $m$. Of course, a particular case of rational first integrals are the polynomial first integrals, i.e. when the denominator of a rational first integral is a non-zero constant.

We note that if a polynomial differential system has a rational first integral, then all the trajectories of this differential system are contained in invariant algebraic curves. The interest in the polynomial differential systems having rational first integrals goes back to Poincaré [13]. But very few progress has been made up to nowadays.

Quadratic systems and its applications have been studied intensively in the last 25 years, and more than one thousand papers have been published about the subject (see for instance $[24,25])$. But the problem of classifying all the integrable quadratic systems remains open. More than that, the problem of classifying all the integrable quadratic having a rational first integral also remains open.

We note that the degenerate quadratic systems can be reduced through a rescaling of the independent variable to the linear differential systems, or to the constant differential systems. So we do not consider them in this work.

The non-degenerate quadratic systems having a polynomial first integral and their phase portraits have been characterized in [8, 9] using normal forms. Later on in [3] applying the invariant theory we provided invariant algebraic conditions in the coefficients of any non-degenerate quadratic system in order to determine if it has or not a polynomial first integral without using any normal form. We obtain that the existence of a polynomial first integral is directly related with the fact that all the roots of a convenient cubic polynomial are rational and negative. The coefficients of this cubic polynomial are invariants related with some geometric properties of the quadratic system.

Since the non-degenerate quadratic systems having a polynomial first integral are classified, now we are interested in the classification of the non-degenerate quadratic systems having a rational first integral $\mathcal{H}$ such that $\mathcal{H}$ and $1 / \mathcal{H}$ are not polynomial. In what follows when we say that $\mathcal{H}$ is a rational first integral we are assuming that $\mathcal{H}$ and $1 / \mathcal{H}$ are not polynomial.

The phase portrait of all non-degenerate quadratic systems having a rational first integral of degree 2 have been characterized in [6] using normal forms. Later on in [1] by identifying every quadratic system with a single point of $\mathbb{R}^{12}$ through its coefficients, and by using first the results of [6] and second the algebraic invariant theory, we classified all the nondegenerate quadratic systems of $\mathbb{R}^{12}$ having a rational first integral of degree 2 . We shown that there are only 24 different topological phase portraits in the Poincaré disc associated to this family of quadratic systems up to a reversal of the sense of their orbits, and we provide a unique representative of every class modulo an affine change of variables and a rescalling of the time variable. Moreover, each one of these 24 representatives is determined by a set of algebraic invariant conditions and each respective first integral is given in an invariant form directly from $\mathbb{R}^{12}$.

Our goal in this paper is to obtain a similar result to [1] for the non-degenerate quadratic systems having a rational first integral of degree 3. Again we shall use a preliminary work, see $[10,11]$, where they are characterized the phase portraits of the non-degenerate quadratic systems having a rational first integral of degree 3 . Then, again using the algebraic invariant theory, we classified all the non-degenerate quadratic systems of $\mathbb{R}^{12}$ having a rational first integral of degree 3. We show that there are only 31 different topological phase portraits in the Poincaré disc associated to this family of quadratic systems up to a reversal of the sense of their orbits, and we provide representatives of every class modulo an affine change of variables and a rescaling of the time variable. Moreover, each one of these 31 representatives is determined by a set of algebraic invariant conditions and we 
provide for it a first integral. These results are stated in our Main Theorem in Section 2 after providing the definitions of the algebraic invariants that we need for the classification.

We must mention that the corrections to the paper [10] made in [11] have been possible due to the present work.

In Section 3 we prove our Main Theorem. We split the proof into several lemmas and divide it according to some subsets to make it clearer.

\section{Basic definitions and Results. Statement of the Main Theorem}

The use of the invariant theory applied to the study of differential polynomial systems (mainly quadratic ones) has allowed to extend the conditions for many families of quadratic systems from the used normal forms to the general system in the parameter space of 12 coefficients. In this direction the works of the Sibirskii school (cf. [21], [23], [14], [4], [7]) have provided the necessary tools for determining the algebraic conditions on general systems to achieve most of the geometric properties of the problem.

We consider real quadratic systems of the form

$$
\begin{aligned}
& \frac{d x}{d t}=p_{0}+p_{1}(x, y)+p_{2}(x, y) \equiv P(x, y), \\
& \frac{d y}{d t}=q_{0}+q_{1}(x, y)+q_{2}(x, y) \equiv Q(x, y),
\end{aligned}
$$

with homogeneous polynomials $p_{i}$ and $q_{i}(i=0,1,2)$ of degree $i$ in $x, y$, i.e.

$$
\begin{array}{ccc}
p_{0}=a_{00}, & p_{1}(x, y)=a_{10} x+a_{01} y, & p_{2}(x, y)=a_{20} x^{2}+2 a_{11} x y+a_{02} y^{2}, \\
q_{0}=b_{00}, & q_{1}(x, y)=b_{10} x+b_{01} y, & q_{2}(x, y)=b_{20} x^{2}+2 b_{11} x y+b_{02} y^{2} .
\end{array}
$$

Let $a=\left(a_{00}, a_{10}, a_{01}, a_{20}, a_{11}, a_{02}, b_{00}, b_{10}, b_{01}, b_{20}, b_{11}, b_{02}\right)$ be the 12 -tuple of the coefficients of system (4) and denote $\mathbb{R}[a, x, y]=\mathbb{R}\left[a_{00}, a_{10}, a_{01}, a_{20}, a_{11}, a_{02}, b_{00}, b_{10}, b_{01}, b_{20}, b_{11}\right.$, $\left.b_{02}, x, y\right]$, i.e. the ring of all polynomials in the variables $a, x$ and $y$.

In order to find affine invariant conditions for determining the class of quadratic systems possessing a polynomial first integral we shall construct the necessary affine invariant polynomials as follows.

We consider the polynomials

$$
\begin{aligned}
& C_{i}(a, x, y)=y p_{i}(a, x, y)-x q_{i}(a, x, y) \in \mathbb{R}[a, x, y], i=0,1,2, \\
& D_{i}(a, x, y)=\frac{\partial}{\partial x} p_{i}(a, x, y)+\frac{\partial}{\partial y} q_{i}(a, x, y) \in \mathbb{R}[a, x, y], i=1,2 .
\end{aligned}
$$

Using the so-called transvectant of index $k$ (see [12]) of two polynomials $f, g \in \mathbb{R}[a, x, y]$

$$
(f, g)^{(k)}=\sum_{h=0}^{k}(-1)^{h}\left(\begin{array}{l}
k \\
h
\end{array}\right) \frac{\partial^{k} f}{\partial x^{k-h} \partial y^{h}} \frac{\partial^{k} g}{\partial x^{h} \partial y^{k-h}}
$$

we construct the following $G L$ - comitants of the second degree with the coefficients of the initial system

$$
\begin{array}{lll}
T_{1}=\left(C_{0}, C_{1}\right)^{(1)}, & T_{2}=\left(C_{0}, C_{2}\right)^{(1)}, & T_{3}=\left(C_{0}, D_{2}\right)^{(1)} \\
T_{4}=\left(C_{1}, C_{1}\right)^{(2)}, & T_{5}=\left(C_{1}, C_{2}\right)^{(1)}, & T_{6}=\left(C_{1}, C_{2}\right)^{(2)} \\
T_{7}=\left(C_{1}, D_{2}\right)^{(1)}, & T_{8}=\left(C_{2}, C_{2}\right)^{(2)}, & T_{9}=\left(C_{2}, D_{2}\right)^{(1)} .
\end{array}
$$

In order to be able to calculate the values of the needed invariant polynomials directly for every canonical system we shall define here a family of $T$-comitants (see [16] for detailed 
definitions) expressed through $C_{i}(i=0,1,2)$ and $D_{j}(j=1,2)$ :

$$
\begin{aligned}
\tilde{A}= & \left(C_{1}, T_{8}-2 T_{9}+D_{2}^{2}\right)^{(2)} / 144, \\
\widetilde{D}= & {\left[2 C_{0}\left(T_{8}-8 T_{9}-2 D_{2}^{2}\right)+C_{1}\left(6 T_{7}-T_{6}\right)-\left(C_{1}, T_{5}\right)^{(1)}+6 D_{1}\left(C_{1} D_{2}-T_{5}\right)-9 D_{1}^{2} C_{2}\right] / 36, } \\
\widetilde{E}= & {\left[D_{1}\left(2 T_{9}-T_{8}\right)-3\left(C_{1}, T_{9}\right)^{(1)}-D_{2}\left(3 T_{7}+D_{1} D_{2}\right)\right] / 72, } \\
\widetilde{F}= & {\left[6 D_{1}^{2}\left(D_{2}^{2}-4 T_{9}\right)+4 D_{1} D_{2}\left(T_{6}+6 T_{7}\right)+48 C_{0}\left(D_{2}, T_{9}\right)^{(1)}-9 D_{2}^{2} T_{4}+288 D_{1} \widetilde{E}\right.} \\
& \left.-24\left(C_{2}, \widetilde{D}\right)^{(2)}+120\left(D_{2}, \widetilde{D}\right)^{(1)}-36 C_{1}\left(D_{2}, T_{7}\right)^{(1)}+8 D_{1}\left(D_{2}, T_{5}\right)^{(1)}\right] / 144, \\
\widetilde{K}= & \left(T_{8}+4 T_{9}+4 D_{2}^{2}\right) / 72, \\
\widetilde{H}= & \left(-T_{8}+8 T_{9}+2 D_{2}^{2}\right) / 72 .
\end{aligned}
$$

We shall use the following invariant polynomials, constructed in [15] and [3]:

$$
\begin{aligned}
& M(a, x, y)=2 \operatorname{Hess}\left(C_{2}(x, y)\right)=\left(C_{2}, C_{2}\right)^{(2)}, \\
& \eta(a)=\operatorname{Discriminant}\left(C_{2}(x, y)\right)=(M, M)^{(2)} / 384, \\
& D(a, x, y)=\widetilde{D}(a, x, y), \\
& K(a, x, y)=4 \widetilde{K}(a, x, y) \equiv \operatorname{Jacob}\left(p_{2}(x, y), q_{2}(x, y)\right), \\
& H(a, x, y)=-4 \widetilde{H}(a, x, y), \\
& \mu_{0}(a)=\operatorname{Discriminant}(K(x, y)) \equiv-(K, K)^{(2)} / 32=\mu(a), \\
& N(a, x, y)=K(a, x, y)+H(a, x, y), \\
& \theta(a)=\operatorname{Discriminant}(N(a, x, y))=-(N, N)^{(2)} / 2, \\
& B_{3}(a, x, y)=\left(C_{2}, \tilde{D}\right)^{(1)}=J a c o b\left(C_{2}, \tilde{D}\right), \\
& B_{2}(a, x, y)=\left(B_{3}, B_{3}\right)^{(2)}-6 B_{3}\left(C_{2}, \tilde{D}\right)^{(3)}, \\
& B_{1}(a)=\operatorname{Res}_{x}\left(C_{2}, \tilde{D}\right) / y^{9}=-2^{-9} 3^{-8}\left(B_{2}, B_{3}\right)^{(4)},
\end{aligned}
$$

as well as the invariant polynomials $\mu_{i}(a, x, y)(i=1, . ., 4)$, constructed in [5] using the invariant $\mu_{0}(a)$ as follows.

Consider the differential operator $\mathcal{L}=x \cdot \mathbf{L}_{2}-y \cdot \mathbf{L}_{1}$ acting on $\mathbb{R}[a, x, y]$, where

$$
\begin{aligned}
& \mathbf{L}_{1}=2 a_{00} \frac{\partial}{\partial a_{10}}+a_{10} \frac{\partial}{\partial a_{20}}+\frac{1}{2} a_{01} \frac{\partial}{\partial a_{11}}+2 b_{00} \frac{\partial}{\partial b_{10}}+b_{10} \frac{\partial}{\partial b_{20}}+\frac{1}{2} b_{01} \frac{\partial}{\partial b_{11}} \\
& \mathbf{L}_{2}=2 a_{00} \frac{\partial}{\partial a_{01}}+a_{01} \frac{\partial}{\partial a_{02}}+\frac{1}{2} a_{10} \frac{\partial}{\partial a_{11}}+2 b_{00} \frac{\partial}{\partial b_{01}}+b_{01} \frac{\partial}{\partial b_{02}}+\frac{1}{2} b_{10} \frac{\partial}{\partial b_{11}}
\end{aligned}
$$

Then

$$
\mu_{i}(a, x, y)=\frac{1}{i !} \mathcal{L}^{(i)}\left(\mu_{0}\right), i=1, . ., 4, \quad \text { where } \quad \mathcal{L}^{(i)}\left(\mu_{0}\right)=\mathcal{L}\left(\mathcal{L}^{(i-1)}\left(\mu_{0}\right)\right)
$$

Remark 1. According to [5] a system (4) is degenerate (i.e. $\operatorname{gcd}(P, Q) \neq 1$ ) if and only if $\mu_{i}=0$ for all $i=0,1, . ., 4$.

For our goal we shall use also the following invariant polynomials, which were constructed and applied in [19],[20], [18] and [17] for characterization of the class of quadratic systems, 
possessing invariant lines of total multiplicity at least 4 (we keep the respective notations):

(8)

$$
\begin{aligned}
& H_{1}(a)=-\left(\left(\left(C_{2}, C_{2}\right)^{(2)}, C_{2}\right)^{(1)}, \tilde{D}\right)^{(3)}, \\
& H_{4}(a)=\left(\left(C_{2}, \tilde{D}\right)^{(2)},\left(C_{2}, D_{2}\right)^{(1)}\right)^{(2)}, \\
& H_{5}(a)=\left(\left(C_{2}, C_{2}\right)^{(2)},(D, D)^{(2)}\right)^{(2)}+8\left(\left(C_{2}, \tilde{D}\right)^{(2)},\left(\tilde{D}, D_{2}\right)^{(1)}\right)^{(2)}, \\
& H_{6}(a, x, y)=16 N^{2}\left(C_{2}, \tilde{D}\right)^{(2)}+H_{2}^{2}\left(C_{2}, C_{2}\right)^{(2)} \\
& H_{9}(a)=-\left(\left((\tilde{D}, \tilde{D})^{(2)}, \tilde{D}\right)^{(1)}, \tilde{D}\right)^{(3)} \\
& H_{10}(a)=\left((N, \tilde{D})^{(2)}, D_{2}\right)^{(1)} \\
& H_{12}(a, x, y)=(\tilde{D}, \tilde{D})^{(2)} \equiv 2 \operatorname{Hess}(\tilde{D}) \\
& N_{1}(a, x, y)=C_{1}\left(C_{2}, C_{2}\right)^{(2)}-2 C_{2}\left(C_{1}, C_{2}\right)^{(2)}, \\
& N_{2}(a, x, y)=D_{1}\left(C_{1}, C_{2}\right)^{(2)}-\left(\left(C_{2}, C_{2}\right)^{(2)}, C_{0}\right)^{(1)}, \\
& N_{5}(a, x, y)=\left[\left(D_{2}, C_{1}\right)^{(1)}+D_{1} D_{2}\right]^{2}-4\left(C_{2}, C_{2}\right)^{(2)}\left(C_{0}, D_{2}\right)^{(1)}
\end{aligned}
$$

Finally we construct here the following new invariant polynomials, which being jointed with the above ones are responsible for the existence of a cubic rational first integral for a quadratic system:

$$
\begin{aligned}
Z_{1}(a, x, y)= & M-2 H \\
Z_{2}(a, x, y)= & {\left[4 H^{2}+3 D_{2}^{2} K+11 H K-5 K^{2}+3 C_{2}\left(C_{2}, K\right)^{(2)}\right] \times } \\
& {\left[-16 H^{2}+3 D_{2}^{2} K-29 H K-25 K^{2}+3 C_{2}\left(C_{2}, K\right)^{(2)}\right] } \\
Z_{3}(a)= & 81\left((\tilde{D}, \tilde{D})^{(2)}, \tilde{K}\right)+4\left[\left(C_{2}, \tilde{D}\right)^{(2)}\right]^{2}, \\
Z_{4}(a)= & 4 D_{1}^{2}\left(9 D_{1}^{2}+72 T_{3}+41 T_{4}\right)+9 T_{4}^{2}-800 D_{1}\left(T_{6}, C_{0}\right)^{(1)}+ \\
+ & 144\left(\left(T_{6}, C_{1}\right)^{(1)}, C_{0}\right)^{(1)}+256\left(\left(T_{8}, C_{0}\right)^{(1)}, C_{0}\right)^{(1)}, \\
Z_{5}(a)= & 36 D_{1}^{4}\left(9 D_{1}^{2}+131 T_{4}+108 T_{3}\right)-4116 D_{1}\left(C_{1}, C_{0} D_{2}\right)^{(2)}\left[2 D_{1}^{2}+9 T_{4}\right]+ \\
+ & 8 D_{1}^{2} T_{3}\left(3687 T_{4}+1258 T_{3}\right)+9 T_{4}^{2}\left(929 D_{1}^{2}+50 T_{4}-400 T_{3}\right)+ \\
+ & 6400\left(D, C_{0}^{2} D_{2}\right)^{(3)}+3200\left(C_{1}, C_{0}^{2}\right)^{(2)}\left(C_{1}, D_{2}^{2}\right)^{(2)}+ \\
+ & 4\left(C_{1}, C_{0} D_{2}\right)^{(2)}\left[7261\left(C_{1}, C_{0} D_{2}\right)^{(2)}-10748 D_{1} T_{3}\right], \\
Z_{6}(a, x, y)= & D_{2} \widetilde{E}-12 C_{2} \tilde{A}-2\left(C_{2}, \widetilde{E}\right)^{(1)}, \\
Z_{7}(a, x, y)= & 2 \widetilde{D}\left(D_{2}^{2}+4 H\right)-3 C_{2}\left[\left(C_{2}, \widetilde{D}\right)^{(2)}+\left(\widetilde{D}, D_{2}\right)^{(1)}+6 \widetilde{F}\right], \\
Z_{8}(a, x, y)= & 8 \widetilde{D}+3 C_{2}\left[2 D_{1}^{2}+\left(C_{1}, C_{1}\right)^{(2)}\right] \\
U_{1}(a)= & 2 H_{4}-H_{10} .
\end{aligned}
$$

Our main result is the following one.

Main Theorem. A non-degenerate quadratic system has a cubic rational first integral if and only if one of the following sets of conditions holds.

A) If $Z_{1} C_{2} \neq 0$ then $\eta \geq 0, M \neq 0, \theta=H_{4}=H_{6}=0$ and, either

$\left.\mathcal{A}_{1}\right) \quad N \neq 0, B_{3}=Z_{2}=0$, or

$\left.\mathcal{A}_{2}\right) \quad N=Z_{3}=0, B_{3} \eta \neq 0$, or

$\left.\mathcal{A}_{3}\right) \quad N=\eta=N_{1}=Z_{4}=0, D \neq 0$.

B) If $Z_{1} \neq 0$ and $C_{2}=0$ then $Z_{5}=0$ and $H_{12} \neq 0$.

C) If $Z_{1}=0$ then $Z_{6}=Z_{7}=0$ and either $M \neq 0$ or $M=Z_{8}=0$ and $D \neq 0$.

Moreover a non-degenerate quadratic system having a cubic rational first integral possesses one of the indicated in Table 1 phase portraits if the respective conditions are fulfilled. These conditions are invariant with respect to the action of the affine group and time rescaling. In the second column of Table 1 the canonical systems obtained via the action of 
this group as well as the corresponding first integrals $\mathcal{H}$ are presented, respectively. Here the invariant polynomials are defined in (6)-(9).

The proof of the Main Theorem is based on the classification of all non-degenerate quadratic systems having a rational first integral of degree three (see $[10,11]$ ), using the constructed explicit normal form. From $[10,11]$ we have the following result.

Proposition 2 (Proposition CRFI $[10,11]$ ). . A non-degenerate quadratic system possesses cubic rational first integral if and only if via an affine transformation and time rescaling it can be written in one of the following forms:

$$
\begin{aligned}
& \left.\dot{x}=4\left(d a-2 b x+c x^{2}\right), \quad \dot{y}=c d+4 b y+4 a y^{2}\right) ; \\
& \dot{x}=a x+b x^{2}, \quad \dot{y}=-2 a y-b x y+c y^{2} ; \\
& \dot{x}=a b x+a c x^{2}, \quad \dot{y}=-2 a b y+b d y^{2} ;
\end{aligned}
$$

\begin{tabular}{|c|c|c|c|c|c|c|}
\hline \multicolumn{4}{|c|}{$\begin{array}{c}\text { Necessary and sufficient } \\
\text { conditions for having } \\
\text { cubic rational first integral }\end{array}$} & $\begin{array}{l}\text { Canonical systems } \\
\text { and first integrals }\end{array}$ & \begin{tabular}{|c} 
Additional \\
conditions for \\
phase portraits
\end{tabular} & \begin{tabular}{|} 
Phase \\
portrait \\
(Fig. 1)
\end{tabular} \\
\hline \multirow{7}{*}{$\begin{array}{l}Z_{1} \neq 0 \\
C_{2} \neq 0\end{array}$} & \multirow{7}{*}{$\begin{array}{c}M \neq 0 \\
\eta \geq 0 \\
\theta=0 \\
H_{4}=0 \\
H_{6}=0\end{array}$} & \multirow{4}{*}{\multicolumn{2}{|c|}{$\begin{array}{c}N \neq 0 \\
B_{3}=Z_{2}=0\end{array}$}} & \multirow{4}{*}{$\begin{array}{c}\dot{x}=c^{2}(2-g)+2 c x+g x^{2} \\
\dot{y}=2(g-1) x y+n y^{2} \\
g \in\{-2,2 / 3,3 / 4,4\} \\
c, n \in\{0,1\} \\
\mathcal{H}=[c(g-2)-g x]^{g-2} \times \\
{[(g-2)(c+x)+n y]^{g} y^{-g}}\end{array}$} & $\begin{array}{c}\eta>0, H_{1} \neq 0 \\
n=1, c=1\end{array}$ & $\mathcal{P}_{1}$ \\
\hline & & & & & $\begin{array}{c}\eta>0, H_{1}=0 \\
n=1, c=0\end{array}$ & $\mathcal{P}_{2}$ \\
\hline & & & & & $\begin{array}{c}\eta=0, L>0 \\
n=0, c=1 \\
g \in\{-2,4\}\end{array}$ & $\mathcal{P}_{3}$ \\
\hline & & & & & $\begin{array}{c}\eta=0, L<0 \\
n=0, c=1 \\
g \in\{2 / 3,4 / 3\}\end{array}$ & $\mathcal{P}_{4}$ \\
\hline & & \multirow{3}{*}{$N=0$} & \multirow{2}{*}{$\begin{array}{c}B_{3} \eta \neq 0 \\
Z_{3}=0\end{array}$} & \multirow{2}{*}{$\begin{array}{c}\dot{x}=a+x^{2}, \dot{y}=4 a+y^{2} \\
a \in\{-1,1\} \\
\mathcal{H}=\frac{4 a x-a y+x^{2} y}{a-x^{2}+x y}\end{array}$} & $\begin{array}{l}H_{1}>0 \\
a=-1\end{array}$ & $\mathcal{P}_{1}$ \\
\hline & & & & & $\begin{array}{c}H_{1}<0 \\
a=1\end{array}$ & $\mathcal{P}_{5}$ \\
\hline & & & $\begin{array}{r}\eta=0, D \neq 0 \\
Z_{4}=N_{1}=0\end{array}$ & $\begin{array}{c}\dot{x}=x^{2}-a^{2}, \dot{y}=y \\
a \in\{1,1 / 4\} \\
\mathcal{H}=\frac{4 a x-a y+x^{2} y}{a-x^{2}+x y}\end{array}$ & - & $\mathcal{P}_{3}$ \\
\hline \multirow{3}{*}{$\begin{array}{l}Z_{1} \neq 0 \\
C_{2}=0\end{array}$} & \multirow{3}{*}{\multicolumn{3}{|c|}{$H_{12} \neq 0, Z_{5}=0$}} & \multirow{3}{*}{$\begin{array}{c}\dot{x}=(x+c)^{2}-1+d y, \\
\dot{y}=x y, \quad d \in\{0,1\} \\
c \in\{1 / 3,2,5\} ; \\
\mathcal{H}=y^{-2} \times \\
{[(c+1)(x+c-1)+d y]^{1-c} \times} \\
{[(c-1)(x+c+1)+d y]^{1+c}}\end{array}$} & $\begin{array}{c}H_{10} \neq 0 \\
d=1\end{array}$ & $\mathcal{P}_{6}$ \\
\hline & & & & & $\begin{array}{c}H_{10}=0, \mu_{2}<0 \\
d=0, c=1 / 3 \\
\end{array}$ & $\mathcal{P}_{7}$ \\
\hline & & & & & $\begin{array}{l}H_{10}=0, \mu_{2}>0 \\
d=0, c \in\{2,5\}\end{array}$ & $\mathcal{P}_{8}$ \\
\hline \multirow{4}{*}{$Z_{1}=0$} & \multicolumn{3}{|c|}{$\begin{array}{c}M=0, D \neq 0 \\
B_{3}=Z_{8}=0\end{array}$} & $\begin{array}{c}\dot{x}=x, \dot{y}=3 y+x^{2} \\
\mathcal{H}=\left(x^{2}+y\right) / x^{3}\end{array}$ & - & $\mathcal{P}_{28}$ \\
\hline & \multirow{3}{*}{\multicolumn{2}{|c|}{$\begin{array}{c}M \neq 0 \\
Z_{6}=Z_{7}=0\end{array}$}} & \multirow{3}{*}{$N=0$} & \multirow{3}{*}{$\begin{array}{c}\dot{x}=x^{2}-\delta^{2} \\
\dot{y}=b+4 \delta y+2 x y \\
\delta, b \in\{0,1\},(\delta, b) \neq(0,0) \\
\mathcal{H}=\frac{b+3 \delta y+3 x y}{(x-\delta)^{3}}\end{array}$} & $\begin{array}{c}H_{6} \neq 0, B_{3}=0, \\
\delta=1, b=0 \\
\end{array}$ & $\mathcal{P}_{29}$ \\
\hline & & & & & $\begin{array}{c}H_{6} \neq 0, B_{3} \neq 0 \\
\delta=1, b=1\end{array}$ & $\mathcal{P}_{30}$ \\
\hline & & & & & $\begin{array}{c}H_{6}=0, \\
\delta=0, b=1\end{array}$ & $\mathcal{P}_{31}$ \\
\hline
\end{tabular}

Table 1 
Table 1 (continued)

\begin{tabular}{|c|c|c|c|c|c|}
\hline \multicolumn{3}{|c|}{$\begin{array}{c}\text { Necessary and sufficient } \\
\text { conditions for having } \\
\text { cubic rational first integral }\end{array}$} & $\begin{array}{l}\text { Canonical systems } \\
\text { and first integrals }\end{array}$ & \multirow{2}{*}{$\begin{array}{c}\text { Additional } \\
\text { conditions for } \\
\text { phase portraits } \\
\eta>0, U_{1}>0 \\
(1, e,-1)\end{array}$} & \multirow{2}{*}{\begin{tabular}{|c|}
$\begin{array}{c}\text { Phase } \\
\text { portrait } \\
\text { (Fig.1) }\end{array}$ \\
$\mathcal{P}_{9}$ \\
\end{tabular}} \\
\hline \multirow{19}{*}{$Z_{1}=0$} & \multirow{19}{*}{$\begin{array}{c}M \neq 0, \\
Z_{6}=Z_{7}=0\end{array}$} & \multirow{19}{*}{$N \neq 0$} & \multirow{19}{*}{$\begin{array}{c}\dot{x}=x y, \\
\dot{y}=b+e x+l x^{2}+3 / 2 y^{2} \\
(b, e, l): \\
b, l \in\{-1,0,1\}, \\
e \in \mathbb{R}^{+} \cup\{0\} ; \\
\mathcal{H}=\left(2 b+3 e x+6 l x^{2}+\right. \\
\left.+3 y^{2}\right) / x^{3}\end{array}$} & & \\
\hline & & & & $\begin{array}{c}\eta>0, U_{1}<0, H_{9}>0 \\
(-1, e,-1), e<2\end{array}$ & $\mathcal{P}_{10}$ \\
\hline & & & & $\begin{array}{c}\eta>0, U_{1}<0 \\
H_{9}<0, H_{4}>0 \\
(-1, e,-1), e>\frac{4}{\sqrt{3}}\end{array}$ & $\mathcal{P}_{11}$ \\
\hline & & & & $\begin{array}{c}\eta>0, U_{1}<0 \\
H_{9}<0, H_{4}<0 \\
(-1, e,-1) \\
2<e<4 / \sqrt{3}\end{array}$ & $\mathcal{P}_{12}$ \\
\hline & & & & $\begin{array}{c}\eta>0, U_{1}<0, \\
H_{9}<0, H_{4}=0, \\
(-1, e,-1), e=\frac{4}{\sqrt{3}}\end{array}$ & $\mathcal{P}_{13}$ \\
\hline & & & & $\begin{array}{c}\eta>0, U_{1}<0, H_{9}=0 \\
(-1,2,-1)\end{array}$ & $\mathcal{P}_{14}$ \\
\hline & & & & $\begin{array}{c}\eta>0, U_{1}=0, H_{4} \neq 0 \\
(0,1,-1)\end{array}$ & $\mathcal{P}_{15}$ \\
\hline & & & & $\begin{array}{c}\eta>0, U_{1}=0, H_{4}=0 \\
(0,0,-1)\end{array}$ & $\mathcal{P}_{16}$ \\
\hline & & & & $\begin{array}{c}\eta<0, U_{1}>0 \\
(-1, e, 1)\end{array}$ & $\mathcal{P}_{17}$ \\
\hline & & & & $\begin{array}{c}\eta<0, U_{1}<0, H_{9}>0 \\
(1, e, 1), e>2\end{array}$ & $\mathcal{P}_{18}$ \\
\hline & & & & $\begin{array}{c}\eta=0, U_{1}<0, H_{9}<0 \\
(1, e, 1), e<2\end{array}$ & $\mathcal{P}_{19}$ \\
\hline & & & & $\begin{array}{c}\eta<0, U_{1}<0, H_{9}=0 \\
(1,2,1) \\
\end{array}$ & $\mathcal{P}_{20}$ \\
\hline & & & & $\begin{array}{c}\eta<0, U_{1}=0, H_{4} \neq 0 \\
(0,1,1)\end{array}$ & $\mathcal{P}_{21}$ \\
\hline & & & & $\begin{array}{c}\eta\left\langle 0, U_{1}=0, H_{4}=0\right. \\
(0,0,1)\end{array}$ & $\mathcal{P}_{22}$ \\
\hline & & & & $\begin{array}{c}\eta=0, H_{4} \neq 0, H_{9}>0 \\
(1,1,0)\end{array}$ & $\mathcal{P}_{23}$ \\
\hline & & & & $\begin{array}{c}\eta=0, H_{4} \neq 0, H_{9}<0 \\
(-1,1,0)\end{array}$ & $\mathcal{P}_{24}$ \\
\hline & & & & $\begin{array}{c}\eta \eta=0, H_{4} \neq 0, H_{9}=0 \\
(0,1,0)\end{array}$ & $\mathcal{P}_{25}$ \\
\hline & & & & $\begin{array}{c}\eta=0, H_{4}=0, H_{6}>0 \\
(1,0,0)\end{array}$ & $\mathcal{P}_{26}$ \\
\hline & & & & $\begin{array}{c}\eta=0, H_{4}=0, H_{6}<0 \\
(-1,0,0)\end{array}$ & $\mathcal{P}_{27}$ \\
\hline
\end{tabular}

$$
\begin{array}{ll}
\dot{x}=a x+b x^{2}-c x y, \quad \dot{y}=-2 y(a+c y) ; & \left(\mathcal{S}_{4}\right)[(18)] \\
\dot{x}=a b x-b x^{2}, \quad \dot{y}=2 a c+a^{2} d-3 c x-3 d x^{2}+2 a b y-3 b x y ; & \left(\mathcal{S}_{5}\right)[(23)] \\
\dot{x}=-2 a d x+2 d x^{2}, \quad \dot{y}=-2 a b+3 b x+c x^{2}-2 a d y+3 d x y ; & \left(\mathcal{S}_{6}\right)[(24)]
\end{array}
$$



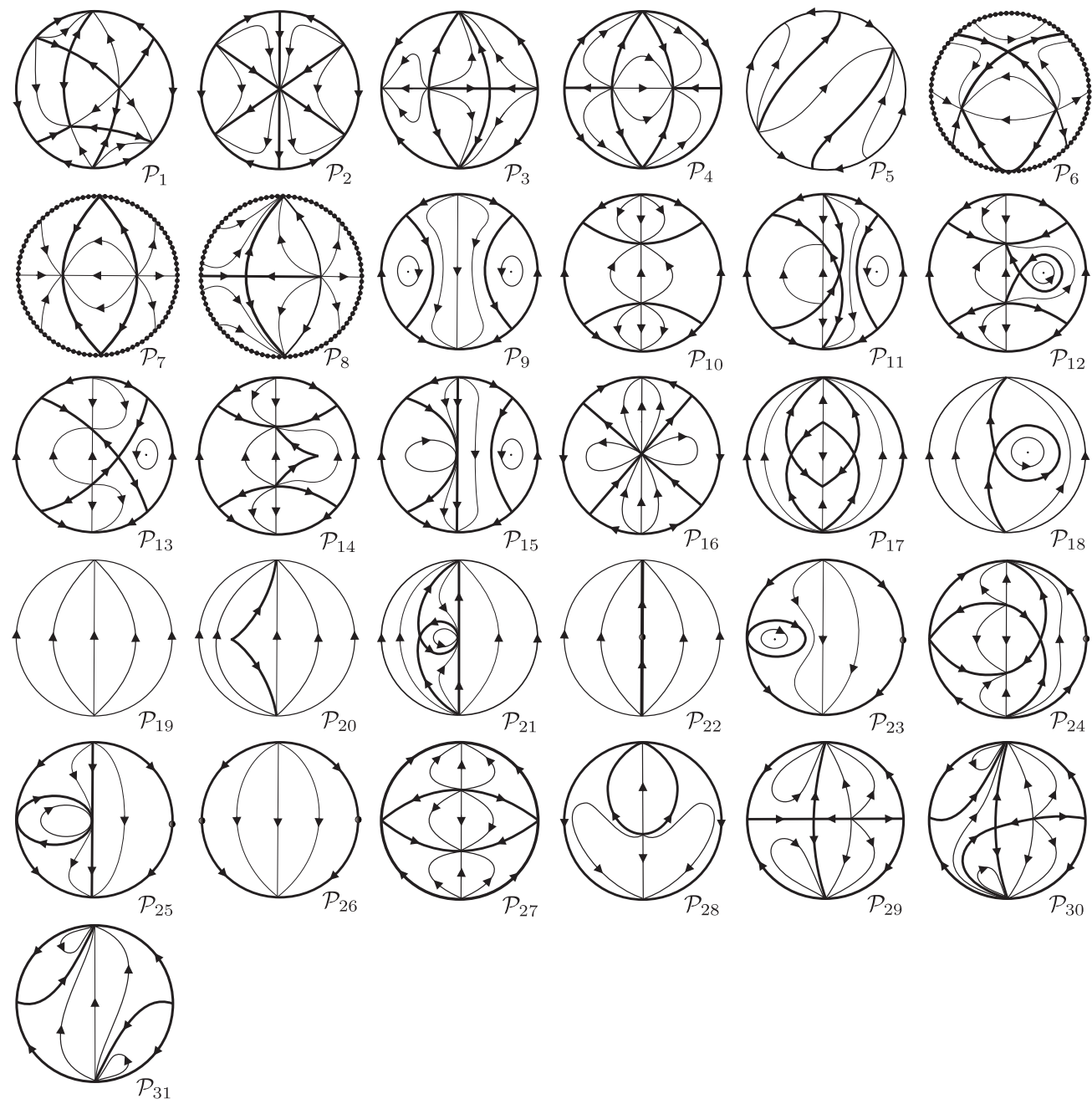

FiguRE 1. The 31 non equivalent topological phase portraits of the planar quadratic polynomial vector field with a rational first integral of degree 3 .

$$
\begin{aligned}
& \dot{x}=2 a x+b x^{2}-2 c x y, \quad \dot{y}=-4 a y+b x y-2 c y^{2} ; \\
& \dot{x}=a b x+a c x^{2}, \quad \dot{y}=c d+c e x+3 b a y+c a x y ; \\
& \dot{x}=2 a b x+2 a c x^{2}, \quad \dot{y}=3 b d+e b x+2 d c x+3 a b y+2 a c x y ; \\
& \dot{x}=6 a c x-6 c x^{2}, \quad \dot{y}=2 a b-3 b x-a d x+4 a c y-6 c x y ; \\
& \dot{x}=2 x(e+a x+b y), \quad \dot{y}=2 f+2 c x+6 e y+2 d x^{2}+4 a x y+3 b y^{2},
\end{aligned}
$$

where the notation $\left(\mathcal{S}_{j}\right)[(k)]$ stands for our compact re-numeration $\left(\mathcal{S}_{j}\right)$ of the families of systems ([(k)]) as they appeared in [10].

The systems $\left(\mathcal{S}_{j}\right)$ for $j=1,2, \ldots, 11$ could be split into the following three classes

$$
\begin{aligned}
& \mathcal{A})\left\{\left(\mathcal{S}_{1}\right),\left(\mathcal{S}_{2}\right), \ldots,\left(\mathcal{S}_{6}\right)\right\}, \\
& \mathcal{B})\left\{\left(\mathcal{S}_{7}\right),\left(\mathcal{S}_{8}\right),\left(\mathcal{S}_{9}\right),\left(\mathcal{S}_{10}\right)\right\}, \\
& \mathcal{C})\left\{\left(\mathcal{S}_{11}\right)\right\}
\end{aligned}
$$

in accordance with some of their common geometrical properties. As it will be proved later on (see Theorems $\mathcal{A}, \mathcal{B}$ and $\mathcal{C}$ in the next section) these properties are described in the next remark. 
Remark 3. A quadratic system belonging to the class either $\mathcal{A})$, or $\mathcal{B})$, or $\mathcal{C}$ ) possesses the following geometrical property.

A system from class $\mathcal{A}$ ) possesses invariant straight lines of total multiplicity 5 (considering the line at infinity and considering their multiplicities).

A system from class $\mathcal{B})$ has the infinity fulfilled of singular points.

$A$ system from class $\mathcal{C})$ in the generic case $\left(b \neq 0\right.$ in $\left.\left(\mathcal{S}_{11}\right)\right)$ possesses a line of symmetry and two singularities (real or complex) located on this line and having zero trace.

\section{Proof of the Main Theorem}

We split the proof of the Main Theorem in three theorems according with the three classes $\mathcal{A}), \mathcal{B}$ ) and $\mathcal{C}$ )

Theorem $\mathcal{A}$. A non-degenerate quadratic system (4) can be written via an affine transformation and a time rescaling as one of the systems from the class $\mathcal{A}$ ) if and only if $\eta \geq 0$, $Z_{1} M \neq 0, \theta=H_{4}=H_{6}=0$ and one of the following sets of conditions holds:

$\left.\mathcal{A}_{1}\right) \quad N \neq 0, B_{3}=Z_{2}=0$;

$\left.\mathcal{A}_{2}\right) \quad N=Z_{3}=0, B_{3} \eta \neq 0$

$\left.\mathcal{A}_{3}\right) \quad N=\eta=N_{1}=Z_{4}=0, D \neq 0$.

In addition any system in class $\mathcal{A})$ has one of the phase portraits $\mathcal{P}_{1}-\mathcal{P}_{5}$ determined by the invariant conditions given in Table 1.

Before proving Theorem $\mathcal{A}$ we shall prove first the following result.

Lemma 4. Assume that for a quadratic system (4) the conditions $\eta \geq 0, Z_{1} M \neq 0$ and $\theta=0$ hold. Then its quadratic part $\left(p_{2}, q_{2}\right)$ could be brought via a linear transformation and a time rescaling into the form

$$
\left(p_{2}, q_{2}\right)=\left(\tilde{g} x^{2}, 2(\tilde{g}-1) x y+\tilde{n} y^{2}\right) \quad\left(\tilde{g} \in \mathbb{R}, \tilde{n} \in\{0,1\},(\tilde{g}-2)^{2}+\tilde{n}^{2} \neq 0\right)
$$

with $\tilde{g} \neq 1$ if $N \neq 0$ and $\tilde{g}=1$ if $N=0$.

Proof. Assume that for the homogeneous quadratic systems

$$
\dot{x}=g x^{2}+2 h x y+k y^{2}, \quad \dot{y}=l x^{2}+2 m x y+n y^{2}
$$

the conditions provided by the lemma are satisfied. Without loss of generality we may assume $k=0$ doing a rotation (if necessary) and then for these systems we calculate

$$
\theta=-64 h\left[l(n-h)^{2}+g m(n-h)-m^{2} n\right] .
$$

We claim that if $\theta=0$ then without loss of generality we may assume $h=0$. Indeed, suppose $h \neq 0$. Then we may assume $g=0$ and $h=1$ due to the transformation $x_{1}=$ $x, y_{1}=g x / 2+h y$, i.e. we get the family of systems

$$
\dot{x}=2 x y, \quad \dot{y}=l x^{2}+2 m x y+n y^{2} .
$$

for which the condition $\theta=0$ yields $l(n-1)^{2}=m^{2} n$. We shall consider two cases: $n \neq 1$ and $n=1$.

If $n \neq 1$ then $l=m^{2} n /(n-1)^{2}$ and via the transformation $x_{1}=m x+(n-1) y, y_{1}=x$, we get systems (11) with $k=h=0$.

Assume now $n=1$. Then the condition $\theta=m^{2}=0$ implies $m=0$, and we arrive to the family of systems

$$
\dot{x}=2 x y, \quad \dot{y}=l x^{2}+y^{2},
$$

for which $\eta=4 l \geq 0$. Then setting $l=u^{2}$ and applying the transformation $x_{1}=u x+y, y_{1}=$ $x$ we again get systems (11) with $k=h=0$.

Thus our claim is proved and in what follows we shall consider the family of systems

$$
\dot{x}=g x^{2}, \quad \dot{y}=l x^{2}+2 m x y+n y^{2}
$$

for which $\theta=0$ and the conditions $Z_{1} M \neq 0$ and $\eta=n^{2}\left[(g-2 m)^{2}-4 l n\right] \geq 0$ have to be fulfilled. 
Assume first $n \neq 0$. In this case we may assume $n=1$ due to a rescaling and then we may set $(g-2 m)^{2}-4 l=u^{2} \geq 0$ (due to $\left.\eta \geq 0\right)$. So we obtain $l=\left[(g-2 m)^{2}-u^{2}\right] / 4$ and via the transformation $x_{1}=x$ and $y_{1}=(2 m-g+u) x / 2+y$, we get the family of systems

$$
\dot{x}=g x^{2}, \quad \dot{y}=2 m x y+y^{2},
$$

for which we calculate $N=4(g-m) m x^{2}$. So if $N \neq 0$ we have $g-m \neq 0$ and via the rescaling $x \rightarrow x /(g-m)$ we get the form (10) with $\tilde{n}=1$ and $\tilde{g} \neq 1$.

Suppose $N=0$, i.e. $m(g-m)=0$. If $m=0$ we have two possibilities: ( $i$ ) if $g \neq 0$ then due to the rescaling $x \mapsto x / g$ we obtain the form (10) with $\tilde{g}=1$ and $\tilde{n}=1$; and (ii) if $g=0$ applying the change $(x, y) \mapsto(y, x)$ we get the same form with $\tilde{g}=1$ and $\tilde{n}=0$.

If $m \neq 0$ then $m=g \neq 0$ and via the transformation $x_{1}=g x$ and $y_{1}=g x+y$ we get the (10) with $\tilde{g}=1$ and $\tilde{n}=1$.

Assume now $n=0$. In this case $M=-8(g-2 m)^{2} x^{2} \neq 0$ and then we may assume $l=0$ due to the transformation $x_{1}=x$ and $y_{1}=-l x+(g-2 m) y$. So we get the family of systems

$$
\dot{x}=g x^{2}, \quad \dot{y}=2 m x y,
$$

for which we calculate $N=4 m(g-m) x^{2}$ and $Z_{1}=8(g-m)(3 m-g) x^{2}$. As $Z_{1} \neq 0$ (i.e. $g-m \neq 0)$ then via the rescaling $x \mapsto x /(g-m)$ we arrive to the form (10) with $\tilde{n}=0$ and $\tilde{g} \neq 1$ if $N \neq 0$, and to the same form with $\tilde{n}=0$ and $\tilde{g}=1$ if $N=0$ (i.e. $m=0$ ). This completes the proof of the lemma.

Proof of Theorem $\mathcal{A}$. Sufficiency. Assuming that for a system (4) the conditions $\eta \geq 0$, $Z_{1} M \neq 0, \theta=H_{4}=H_{6}=0$ hold, we shall consider step by step each set of the conditions $\mathcal{A}_{i}$ ) for $i=1,2,3$.

Case $\left.\mathcal{A}_{1}\right)$. As $N \neq 0$ according to Lemma 4 we can consider the quadratic parts of this system being in the form (4) with $\tilde{g} \neq 1$. Therefore due to an additional translation we get the family of systems

$$
\begin{gathered}
\dot{x}=a+c x+d y+g x^{2}, \quad \dot{y}=b+2(g-1) x y+n y^{2}, \\
n \in\{0,1\}, \quad g \neq 1, \quad(g-2)^{2}+n^{2} \neq 0,
\end{gathered}
$$

for which we calculate

$$
Z_{2}=256(g-1)^{2}(g-4)(g+2)(3 g-4)(3 g-2) x^{8},
$$

and

$$
\begin{array}{cl}
\text { Coeff }\left[B_{3}, x^{4}\right]=12 b(g-2)(-1+g)^{2}, & \text { Coeff }\left[B_{3}, x^{3} y\right]=24 b(g-1)^{2} n, \\
\text { Coeff }\left[B_{3}, x y^{3}\right]=6 d^{2} g(g-2), & \text { Coeff }\left[B_{3}, y^{4}\right]=3 d^{2} g n .
\end{array}
$$

Hence considering (13) and the fact that the condition $Z_{2}=0$ implies $g \neq 0$, we obtain that $B_{3}=0$ yields $b=d=0$. Then calculations yield

$$
\begin{aligned}
B_{3} & =-3\left(4 a-2 c^{2}+c^{2} g\right) n x^{2} y^{2}, \\
\text { Coeff }\left[H_{6}, x^{6}\right] & =2048(2-g)(g-1)^{4}\left(4 a-2 c^{2}+c^{2} g\right) .
\end{aligned}
$$

So considering (13) the conditions $B_{3}=H_{6}=0$ yield $4 a-2 c^{2}+c^{2} g=0$, and this implies $H_{6}=H_{4}=0$.

To construct a simpler canonical form we replace $c \mapsto 2 c$ and taking into consideration the relation above and $Z_{2}=0$ we get the families of systems

$$
\dot{x}=c^{2}(2-g)+2 c x+g x^{2}, \quad \dot{y}=2(g-1) x y+n y^{2},
$$

where $n \in\{0,1\}, g \in\{-2,2 / 3,4 / 3,4\}, c \in\{0,1\}$ (due to a rescaling) and $(c, n) \neq(0,0)$ (otherwise we get degenerate systems). These systems possess the first integral

$$
\mathcal{H}=[c(g-2)-g x]^{g-2}[(g-2)(c+x)+n y]^{g} y^{-g},
$$

and easily we see that $\mathcal{H}$ is a cubic rational first integral if and only if $g \in\{-2,2 / 3,4 / 3,4\}$.

We observe that the family of systems (15) possesses the invariant straight lines

$$
x+c=0, \quad c(g-2)-g x=0, \quad y=0, \quad(g-2)(c+x)+n y=0 .
$$


So this family of systems possess invariant straight lines (including the line at infinity) with total multiplicity at least five (see [15] for detailed definitions). In fact as it will be shown later on, the conditions provided by Theorem $\mathcal{A}$ imply the existence of invariant straight lines of multiplicity 5 . Thus in order to determine the phase portraits which could be realized for the family (15) we could apply the respective results from [17]. We observe that for systems (15) we have

$$
\begin{aligned}
& B_{3}=\theta=H_{6}=0, \quad \eta=n^{2}(g-2)^{2}, \quad \mu_{0}=g^{2} n^{2}, \quad K=4 g x[(g-1) x+n y], \\
& N=4(g-1) x^{2}, \quad H_{1}=2304 c^{2}(g-2)^{2}(g-1)^{2} n^{2}, \quad g \in\{-2,2 / 3,4 / 3,4\} .
\end{aligned}
$$

Suppose that $\eta>0$. Then $n \neq 0$ (i.e. $n=1$ ) and this implies $\mu_{0} \neq 0$. So according to $\left[17\right.$, Table 5] the phase portrait of a system (15) corresponds to $\mathcal{P}_{1}$ (see Figure 1) if $H_{1} \neq 0$ (i.e. $c=1$ ), and corresponds to $\mathcal{P}_{2}$ if $H_{1}=0$ (i.e. $c=0$ ).

It remains to note that when $n=1$ we could consider $g \in\{-2,4\}$ and the other cases can be reduced to these two. To show this it is sufficient to construct the two transformations (we use the notation $(c, g, n)$ for parameters):

$$
\begin{aligned}
& x_{1}=-3(x+4 c), y_{1}=-4 x+y-4 c \text { then }[(15)]_{(c,-2,1)} \text { implies } \Rightarrow[(15)]_{(\tilde{c}, 2 / 3,1)} ; \\
& x_{1}=3(x-2 c), \quad y_{1}=2 x+y+2 c \text { then }[(15)]_{(c, 4,1)} \text { implies } \Rightarrow[(15)]_{(\tilde{c}, 4 / 3,1)} .
\end{aligned}
$$

Assume now $\eta=0$. We get $n=0$ (then $c \neq 0$, i.e. $c=1)$ and this implies $\mu_{0}=0$. In this case for systems (15) we calculate: $L=8 g(2-g) x^{2} \neq 0$ for the given values of the parameter g. As $K N \neq 0$, then according to [17] the phase portrait of a system (15) corresponds to $\mathcal{P}_{3}$ if $L>0$ (i.e. $g \in\{2 / 3,4 / 3\}$ ), and corresponds to $\mathcal{P}_{4}$ if $L<0$ (i.e. $g \in\{-2,4\}$ ).

Case $\mathcal{A}_{2}$ ). Then for system (4) the condition $N=0$ holds. According to Lemma 4 we could consider its quadratic parts being in the form (4) with $\tilde{g}=1$. Therefore due to an additional translation we get the family of systems

$$
\dot{x}=a+d y+x^{2}, \quad \dot{y}=b+e x+f y+n y^{2}, \quad n \in\{0,1\},
$$

for which we have $H_{4}=96\left(d^{2}+e^{2} n^{4}\right)$ and $\eta=n^{2}$. So the condition $H_{4}=0$ yields $d=e n=0$, and since $\eta \neq 0$ (i.e. $n=1$ ) this implies $e=0$. Hence applying the additional translation $y \mapsto y-f / 2$, we get the family of systems

$$
\dot{x}=a+x^{2}, \quad \dot{y}=b+y^{2},
$$

for which we calculate

$$
B_{3}=12(b-a) x^{2} y^{2}, \quad Z_{3}=2304(a-4 b)(4 a-b) .
$$

The condition $Z_{3}=0$ yields $(a-4 b)(4 a-b)=0$, and due to the change $(x, y, a, b) \mapsto$ $(y, x, b, a)$ we may assume $b=4 a \neq 0\left(\right.$ as $\left.B_{3} \neq 0\right)$. Thus we arrive to the systems

$$
\dot{x}=a+x^{2}, \quad \dot{y}=4 a+y^{2},
$$

with $a \in\{-1,1\}$ (due to a rescaling). It could be checked directly that the cubic rational function

$$
\mathcal{H}=\frac{4 a x-a y+x^{2} y}{a-x^{2}+x y}
$$

is a first integral for these systems. We observe that systems (17) also possess invariant straight lines of total multiplicity five (the affine lines being $x^{2}+a=0$ and $y^{2}+4 a=0$ ).

It remains to observe that for systems (17) we have

$$
\eta=1>0, B_{2}=N=H_{4}=0, B_{3}=36 a x^{2} y^{2} \neq 0, H_{5}=24576 a^{2}>0, H_{1}=-5760 a,
$$

and according to [17] we get the phase portrait $\mathcal{P}_{1}$ if $H_{1}>0$ (i.e. $a=-1$ ), and $\mathcal{P}_{5}$ if $H_{1}<0$ (i.e. $a=1$ ).

Case $\left.\mathcal{A}_{3}\right)$. Since besides the condition $N=0$ we have also $\eta=0$, then the condition $H_{4}=0$ for systems (17) yields $d=n=0$. So we get the family of systems

$$
\dot{x}=a+x^{2}, \quad \dot{y}=b+e x+f y,
$$


for which we calculate

$$
D=-f^{2} x^{2} y, N_{1}=8 e x^{4}, Z_{4}=-256\left(a+f^{2}\right)\left(16 a+f^{2}\right) .
$$

The condition $N_{1}=0$ yields $e=0$, whereas the condition $D \neq 0$ implies $f \neq 0$, and then we could assume $f=1$ (due to a rescaling), and $b=0$ (due to a translation). As the condition $Z_{4}=0$ implies $a<0$ we replace $a \mapsto-a^{2}$, and then considering the condition $Z_{4}=0$ and $f=1$, we arrive to systems

$$
\dot{x}=x^{2}-a^{2}, \quad \dot{y}=y, \quad a \in\{1,1 / 4\},
$$

possessing the first integral $\mathcal{H}=\frac{(a+x) y^{2 a}}{a-x}$. Clearly both values of $a \in\{1,1 / 4\}$ lead to cubic rational function $\mathcal{H}$.

It remains to note that for systems (19) we have

$$
B_{3}=N=\eta=H=N_{1}=0, M=-8 x^{2} \neq 0, N_{5}=64 a^{2} x^{2}>0, N_{2}=4\left(1-4 a^{2}\right) x \neq 0,
$$

and according to [17] for both values of $a \in\{1,1 / 4\}$ we obtain the phase portrait which is topologically equivalent to $\mathcal{P}_{3}$. We remark that by [17] the above conditions imply also the existence of the invariant straight lines of total multiplicity five for these systems.

Necessity. Assume that a system (4), which possesses a cubic rational first integral, could be written via an affine transformation and time rescaling as a system from the class $\mathcal{A}$ ). We claim that for this system one of the sets of conditions provided in Theorem $\mathcal{A}$ must hold. To prove this claim we shall consider step by step each family $\left(\mathcal{S}_{i}\right)(i=1,2, \ldots, 6)$ of non-degenerate quadratic systems from the class $\mathcal{A}$ ) and calculate the respective invariant polynomials.

$$
\begin{gathered}
\text { Systems }\left(\mathcal{S}_{1}\right): \quad \dot{x}=4\left(d a-2 b x+c x^{2}\right), \quad \dot{y}=c d+4 b y+4 a y^{2}, \text { then } \\
\theta=H_{4}=H_{6}=N=Z_{3}=0, M=-128\left(c^{2} x^{2}-a c x y+a^{2} y^{2}\right), \eta=256 a^{2} c^{2}, \\
Z_{1}=-128(c x-a y)^{2}, B_{3}=2304 a c\left(b^{2}-a c d\right) x^{2} y^{2} .
\end{gathered}
$$

For these systems the condition $a\left(b^{2}-a c d\right) \neq 0$ must hold, otherwise we get systems with rational first integral of degree less than three. Then evidently $Z_{1} M \neq 0$.

If $\eta \neq 0$ then $B_{3} \neq 0$, and we get the conditions $\mathcal{A}_{2}$ ). If $\eta=0$ we have $c=0$, and then we obtain

$$
N_{1}=Z_{4}=0, D=256 a b^{2} x y^{2},
$$

and as $a b \neq 0$ (see the above reason) this leads to the set of conditions $\mathcal{A}_{3}$ ).

Systems $\left(\mathcal{S}_{2}\right): \quad \dot{x}=a x+b x^{2}, \quad \dot{y}=-2 a y-b x y+c y^{2}$, then

$$
\begin{gathered}
\theta=H_{4}=H_{6}=B_{3}=Z_{2}=0, M=-8\left(4 b^{2} x^{2}-2 b c x y+c^{2} y^{2}\right), \\
\eta=4 b^{2} c^{2}, N=-3 b^{2} x^{2}, Z_{1}=-2\left(15 b^{2} x^{2}-12 b c x y+4 c^{2} y^{2}\right) .
\end{gathered}
$$

We observe that $M Z_{1} \neq 0$, otherwise we obtain $b=c=0$ that leads to linear systems.

If $N \neq 0$ then evidently we get the conditions $\mathcal{A}_{1}$ ). Assuming $N=0$ (i.e. $b=0$ and then $c \neq 0)$, we obtain

$$
\eta=N=N_{1}=Z_{4}=0, D=a^{2} c x y^{2} \neq 0,
$$

and this leads to the set of conditions $\mathcal{A}_{3}$ ).

$$
\begin{aligned}
\text { Systems }\left(\mathcal{S}_{3}\right): \quad & \dot{x}=a b x+a c x^{2}, \quad \dot{y}=-2 a b y+b d y^{2}, \text { then } \\
\theta= & H_{4}=H_{6}=N=Z_{3}=0, M=-8\left(a^{2} c^{2} x^{2}-a b c d x y+b^{2} d^{2} y^{2}\right), \\
& \eta=a^{2} b^{2} c^{2} d^{2}, Z_{1}=-8(a c x-b d y)^{2}, B_{3}=-9 a^{3} b^{3} c d x^{2} y^{2} .
\end{aligned}
$$

So $M Z_{1} \neq 0$, otherwise we obtain $a c=b d=0$ and we get linear systems.

If $\eta \neq 0$ then $B_{3} \neq 0$, and evidently we have the set of conditions $\mathcal{A}_{2}$ ). If $\eta=0$ since $a b \neq 0$ (otherwise we get degenerate systems), we obtain $c d=0$. Then we calculate

$$
N_{1}=Z_{4}=0, D=-a^{2} b^{2} x y(4 a c x-b d y),
$$


and as $c^{2}+d^{2} \neq 0$ (if $c=d=0$ we get linear systems), we obtain $D \neq 0$ and this leads to the set of conditions $\mathcal{A}_{3}$ ).

$$
\begin{aligned}
\text { Systems }\left(\mathcal{S}_{4}\right): & \dot{x}=a x+b x^{2}-c x y, \quad \dot{y}=-2 y(a+c y), \text { then } \\
\theta & =H_{4}=H_{6}=B_{3}=Z_{2}=0, M=-8\left(b^{2} x^{2}+b c x y+c^{2} y^{2}\right), \\
\eta & =b^{2} c^{2}, \quad N=3 c^{2} y^{2}, Z_{1}=-2\left(4 b^{2} x^{2}+12 b c x y+3 c^{2} y^{2}\right) .
\end{aligned}
$$

We observe that $M Z_{1} \neq 0$, otherwise we obtain $b=c=0$, that leads to linear systems. So if $N \neq 0$, then and we get the set of conditions $\mathcal{A}_{1}$ ).

Assuming $N=0$ we obtain $c=0$, and then for systems $\left(\mathcal{S}_{4}\right)$ we calculate

$$
N_{1}=Z_{4}=0, D=-4 a^{2} b x^{2} y
$$

and as $a b \neq 0$ (otherwise we get either linear or degenerate systems), we obtain $D \neq 0$ and this leads to the set of conditions $\mathcal{A}_{3}$ ).

$$
\begin{gathered}
\text { Systems }\left(\mathcal{S}_{5}\right): \quad \dot{x}=a b x-b x^{2}, \dot{y}=2 a c+a^{2} d-3 c x-3 d x^{2}+2 a b y-3 b x y, \text { then } \\
\theta=H_{4}=H_{6}=B_{3}=Z_{2}=0, M=-32 b^{2} x^{2}, \\
N=-3 b^{2} x^{2}, Z_{1}=-14 b^{2} x^{2} .
\end{gathered}
$$

As $b \neq 0$ (otherwise systems become degenerate), we have $Z_{1} M N \neq 0$ and this leads to the set of conditions $\left.\mathcal{A}_{1}\right)$.

Systems $\left(\mathcal{S}_{6}\right): \quad \dot{x}=-2 a d x+2 d x^{2}, \quad \dot{y}=-2 a b+3 b x+c x^{2}-2 a d y+3 d x y$, then

$$
\begin{gathered}
\theta=H_{4}=H_{6}=B_{3}=Z_{2}=0, M=-8 d^{2} x^{2}, \\
N=3 d^{2} x^{2}, Z_{1}=10 d^{2} x^{2} .
\end{gathered}
$$

As $d \neq 0$ (otherwise systems become degenerate), we have $Z_{1} M N \neq 0$ and this leads to the set of conditions $\left.\mathcal{A}_{1}\right)$.

As all the possible cases are examined this completes the proof of the theorem.

Theorem $\mathcal{B}$. A non-degenerate quadratic system (4) can be written via an affine transformation and a time rescaling as one of the systems of the class $\mathcal{B})$ if and only if $C_{2}=Z_{5}=0$ and $H_{12} \neq 0$. In addition any system in class $\left.\mathcal{B}\right)$ has one of the phase portraits $\mathcal{P}_{6}-\mathcal{P}_{8}$ determined by the invariant conditions given in Table 1.

Proof. Necessity. Assume that a quadratic system having a cubic rational first integral belongs to the class $\mathcal{B}$ ). Straightforward calculations show that for each of the families $\mathcal{S}_{7}$, $\mathcal{S}_{8}, \mathcal{S}_{9}$ and $\mathcal{S}_{10}$ we have $C_{2}=Z_{5}=0$. The condition $C_{2}=0$ means that any system from this class has the infinity fulfilled of singular points. Moreover for these systems we obtain

$\left(\mathcal{S}_{7}\right)$ has $H_{12}=-4608 a^{4}\left(b^{2} x^{2}+b c x y+c^{2} y^{2}\right), \quad\left(\mathcal{S}_{8}\right)$ has $H_{12}=-288 a^{6} b^{4} c^{2} x^{2}$,

$\left(\mathcal{S}_{9}\right)$ has $H_{12}=-288 a^{6} b^{4} c^{2} x^{2}$,

$\left(\mathcal{S}_{10}\right)$ has $H_{12}=-2^{11} 3^{2} a^{4} c^{6} x^{2}$

It obviously could be checked that for each of the above families of systems the condition $H_{12}=0$ leads to either degenerate, or linear systems, or to systems with rational first integral of degree 2 . And the last situation could happens only for family $\left(\mathcal{S}_{8}\right)$ when $b=0$. Indeed a first integral of this family (see [10]) is

$$
\mathcal{H}=x^{-3}\left[2 b c d+3 c(c d+b e) x+6 c^{2} e x^{2}+6 a(b+c x)^{2} y\right],
$$

which for $b=0$ becomes quadratic. Thus the necessity is proved.

Sufficiency. Assuming that the conditions provided by the theorem are verified for a quadratic system (4) we shall show that it possesses a cubic rational first integral. As $C_{2}=0$ according to [19] any such system could be written via an affine transformation and time rescaling to one of the nine canonical systems $\left(C_{2} \cdot j\right), j=1,2, \ldots, 9$ (see [19, Table 1]). Among these systems only the systems corresponding to $j=1,2,3,5,6,7$ could satisfy the condition $H_{12} \neq 0$. 
Straightforward calculations show that the condition $Z_{5}=0$ could be satisfied only for two systems in this class, and namely, for systems

$$
\dot{x}=(x+c)^{2}-1+y, \quad \dot{y}=x y,
$$

if $H_{10} \neq 0$ and for systems

$$
\dot{x}=(x+c)^{2}-1, \quad \dot{y}=x y,
$$

if $H_{10}=0$. We observe that these two families of systems could be joint into the family

$$
\dot{x}=(x+c)^{2}-1+d y, \quad \dot{y}=x y,
$$

with $d \in\{0,1\}$ and possess the first integral

$$
\mathcal{H}=y^{-2}[(c+1)(x+c-1)+d y]^{1-c}[(c-1)(x+c+1)+d y]^{1+c} .
$$

For these systems we calculate

$$
Z_{5}=2304\left(c^{2}-25\right)\left(c^{2}-4\right)\left(9 c^{2}-1\right)=0 \quad \text { then } \quad c \in\{ \pm 1 / 3, \pm 2, \pm 5\} .
$$

It can be checked that any indicated value of the parameter $c$ leads to a cubic rational first integral. However we could minimize this set of values.

Remark 5. Due to the change $(x, y, t) \mapsto(-x, y,-t)$ we may assume $c \in\{1 / 3,2,5\}$ in both systems $\left(C_{2} .1\right)$ and $\left(C_{2} .5\right)$.

Moreover in systems $\left(C_{2} .1\right)$ we may assume $c=2$ due to the transformations

$$
\begin{aligned}
& x_{1}=-\frac{1}{3} x-\frac{4}{3}, y_{1}=\frac{4}{9} x+\frac{1}{9} y+\frac{8}{3}, t_{1}=-3 t, \text { system }\left.\left(C_{2} \cdot 1\right)\right|_{c=5} \text { pass to }\left.\left(C_{2} \cdot 1\right)\right|_{c=1 / 3} ; \\
& x_{1}=-\frac{1}{2} x-3, y_{1}=\frac{3}{2} x+\frac{1}{4} y+6, t_{1}=-2 t, \text { system }\left.\left(C_{2} \cdot 1\right)\right|_{c=5} \text { pass to }\left.\left(C_{2} \cdot 1\right)\right|_{c=2} .
\end{aligned}
$$

In accordance with this remark we get the respective canonical system of Table 1 . Moreover, if $H_{10}=0$, then according to [19] these systems possesses a phase portrait topologically equivalent to $\mathcal{P}_{6}$.

Assume $H_{10}=0$, i.e. we consider $\left(C_{2} .5\right)$, for which by Remark 5 we have $c \in\{1 / 3,2,5\}$. For these systems we calculate $\mu_{2}=\left(c^{2}-1\right) x^{2}$, and according to [19] there phase portraits are $\mathcal{P}_{7}$ if $\mu_{2}<0$ (i.e. $c=1 / 3$ ), and $\mathcal{P}_{8}$ if $\mu_{2}>0$ (i.e. $c=2,5$ ). We note that in the last case systems $\left(C_{2} .5\right)$ with $c=2$ and with $c=5$ are located on different affine orbits. Note that for systems $\left(C_{2} .1\right)$ and $\left(C_{2} .5\right)$ we have $Z_{1} \neq 0$. This completes the proof of the theorem.

Theorem $\mathcal{C}$. A non-degenerate quadratic system (4) can be written via an affine transformation and a time rescaling as one of the systems of the class $\mathcal{C}$ ) if and only if $Z_{1}=Z_{6}=$ $Z_{7}=0$ and either $M \neq 0$, or $M=B_{3}=Z_{8}=0$ and $D \neq 0$. In addition any system in class $\mathcal{C})$ has one of the phase portraits $\mathcal{P}_{9}-\mathcal{P}_{31}$ determined by the invariant conditions given in Table 1.

Before proving Theorem $\mathcal{C}$ we shall prove first the following result.

Lemma 6. Assume that for a quadratic system (4) the condition $Z_{1}=0$ holds. Then its quadratic part $\left(p_{2}, q_{2}\right)$ could be brought via a linear transformation and a time rescaling to one of the form below if and only if the respective conditions hold:

$$
\begin{array}{lll}
\text { (i) } \quad\left(p_{2}, q_{2}\right)=\left(x y, l x^{2}+3 / 2 y^{2}\right)(l \in\{0, \pm 1\}) & \Leftrightarrow \quad N \neq 0 ; \\
\text { (ii }) \quad\left(p_{2}, q_{2}\right)=\left(x^{2}, 2 x y\right) & \Leftrightarrow \quad N=0, M \neq 0 ; \\
\text { (iii }) \quad\left(p_{2}, q_{2}\right)=\left(0, x^{2}\right) & \Leftrightarrow \quad N=M=0 .
\end{array}
$$

Proof. Necessity. Assuming that a system (4) has the quadratic parts of the form $(i)$ (respectively $($ ii $) ;($ iii $)$ ) calculations yield: $Z_{1}=0$ and $N=2\left(2 l x^{2}+y^{2}\right)$ (respectively $\left.Z_{1}=N=0, M=-8 x^{2} ; Z_{1}=N=M=0\right)$. This proves the necessity.

Sufficiency. We consider the homogeneous quadratic systems (11) and without loss of generality we may assume $k=0$ doing a rotation (if necessary). 
Assume $h \neq 0$. Then we may consider $g=0$ and $h=1 / 2$ due to the transformation $x_{1}=x, y_{1}=g x+2 h y$, and we get the canonical form

$$
\dot{x}=x y, \quad \dot{y}=l x^{2}+2 m x y+n y^{2} .
$$

For these systems we calculate Coefficient $\left[Z_{1}, y^{2}\right]=2(3-2 n)(2 n-1)$, and we shall consider two cases $n=3 / 2$ and $n=1 / 2$.

Assume first $n=3 / 2$. Then calculations yield

$$
Z_{1}=-8 m x(3 m x+2 y), N=4\left(l-m^{2}\right) x^{2}+4 m x y+2 y^{2} .
$$

We observe that $N \neq 0$. The condition $Z_{1}=0$ yields $m=0$, and we get the canonical form $(i)$. Moreover, if $l \neq 0$, then we may assume $l \in\{-1,1\}$ due to the additional rescaling $x \mapsto|l|^{-1 / 2} x$.

If $n=1 / 2$ we have

$$
Z_{1}=-8\left(2 l+3 m^{2}\right) x^{2}, N=-4 m x(m x-y),
$$

and the relation $Z_{1}=0$ implies $l=-3 m^{2} / 2$. Hence we get the family of systems

$$
\dot{x}=x y, \quad \dot{y}=-3 m^{2} x^{2} / 2+2 m x y+y^{2} / 2,
$$

which in the case $N \neq 0$ (i.e. $m \neq 0$ ) could be brought to the form $(i)$ with $l=-1$ via the transformation $x_{1}=(-3 m x+y) / \sqrt{2}, y_{1}=m x+y$ and $t_{1}=t / 2$. If $N=0$ (i.e. $m=0$ ) then via the change $(x, y, t) \mapsto(y, x, 2 t)$, we get the form $(i i)$ for which $M=-8 x^{2} \neq 0$.

Assume now $h=0$. For systems (11) with $k=h=0$ we obtain Coefficient $\left[Z_{1}, y^{2}\right]=$ $-8 n^{2} y^{2}$, and the condition $Z_{1}=0$ implies $n=0$. Thus we get the family of systems

$$
\dot{x}=g x^{2}, \quad \dot{y}=l x^{2}+2 m x y,
$$

for which calculations yield

$$
Z_{1}=8(g-m)(3 m-g) x^{2}, N=4(g-m) m x^{2}, M=-8(g-2 m)^{2} x^{2} .
$$

If $N \neq 0$ then we obtain $g=3 m \neq 0$, and via the transformation $x_{1}=-l x+m y, y_{1}=2 m x$ we get the form $(i)$ with $l=0$.

Assume $N=0$. If $M \neq 0$ then we get $g=m \neq 0$, and obviously via the change $x_{1}=m x$, $y_{1}=l x+m y$ we obtain form (ii). If $M=0$ then $g=m=0$, and evidently due to a rescaling we get the form $(i i i)$. This completes the proof of the lemma.

Proof of Theorem $\mathcal{C}$. Necessity. We shall consider the family $\left(\mathcal{S}_{11}\right)$ (which corresponds to the family of systems (20) from [10]):

$$
\dot{x}=2 x(e+a x+b y), \quad \dot{y}=2 f+2 c x+6 e y+2 d x^{2}+4 a x y+3 b y^{2},
$$

for which $Z_{1}=Z_{6}=Z_{7}=0$ and $M=-16\left(2 a^{2}-3 b d\right) x^{2}-16 a b x y-8 b^{2} y^{2}$. We observe that the relation $M=0$ is equivalent to $b=a=0$, and in this case $B_{3}=Z_{8}=0$ and $D=72 d e^{2} x^{3}$. Clearly $D \neq 0$, otherwise we get either degenerate (if $e=0$ ), or linear (if $d=0$ ) systems. This proves the necessity of the conditions provided in Theorem $\mathcal{C}$.

Sufficiency. In order to prove the sufficiency of these conditions we shall use the canonical forms of quadratic parts provided by Lemma 6 . For systems (24) we calculate $N=8 b\left(2 d x^{2}+\right.$ $\left.2 a x y+b y^{2}\right)$.

Case $N \neq 0$. Then $b \neq 0$, and via the transformation $x_{1}=x, y_{1}=a x+b y+e, t_{1}=2 t$ the 6 -parameter family of systems (24) will be brought to the 3 -parameter family

$$
\dot{x}=x y, \quad \dot{y}=b+e x+l x^{2}+3 / 2 y^{2},
$$

where $b, e$ and $l$ are some new real parameters. We may consider $b, l \in\{-1,0,1\}$ due to the rescaling $(x, y, t) \mapsto\left(\alpha^{-1 / 2} \beta^{1 / 2} x, \beta^{1 / 2} y, \beta^{-1 / 2} t\right)$, where $\alpha=|l|$ (respectively $\alpha=1$ ) if $l \neq 0$ (respectively $l=0$ ), and $\beta=|b|$ (respectively $\beta=1$ ) if $b \neq 0$ (respectively $b=0$ ). Moreover we may assume $e \geq 0$ due to the change $x \mapsto-x$. We note that a first integral for this family is the cubic rational function $\mathcal{H}=\left(2 b+3 e x+6 l x^{2}+3 y^{2}\right) / x^{3}$. 
Consider now a generic quadratic system (4). According to Lemma 6 as $N \neq 0$ and $Z_{1}=0$, applying an additional translation (if necessary) this system can be written as

$$
\dot{x}=a+x y, \quad \dot{y}=b+e x+f y+l x^{2}+3 / 2 y^{2},
$$

where $l \in\{-1,0,1\}$. For these systems the condition $Z_{6}=3 f y\left(2 c l x^{2}+y^{2}\right) / 2=0$ yields $f=0$, and then the condition $Z_{7}=24 a y\left(6 l x^{2}-y^{2}\right)\left(2 l x^{2}+y^{2}\right)$ is equivalent to $a=0$. Thus we get the family of systems (25) and this proves the sufficiency of the conditions in this case.

Case $N=0$. Then $b=0$ and for systems (24) we calculate $M=-32 a^{2} x^{2}$.

Subcase $M \neq 0$. Then $a \neq 0$, and via the transformation

$$
x_{1}=x+\frac{e}{2 a}, \quad y_{1}=\frac{d}{a} x+y+\frac{a c-2 d e}{2 a^{2}}, \quad t_{1}=2 a t,
$$

systems (24) (with $b=0$ and $a \neq 0$ ) will be brought to the family of systems

$$
\dot{x}=x^{2}-\delta^{2}, \quad \dot{y}=b+4 \delta y+2 x y,
$$

having the first integral $\mathcal{H}=(b+3 \delta y+3 x y) /(x-\delta)^{3}$. We may assume $\delta, b \in\{0,1\}$ due to the rescaling $(x, y, t) \mapsto(\delta x, y / \delta, t / \delta)$ if $\delta \neq 0$, and $y \mapsto b y$ if $b \neq 0$.

Consider now a quadratic system (4). As $N=Z_{1}=0$ and $M \neq 0$ according to Lemma 6 after an additional translation (if necessary) this system can be written as

$$
\dot{x}=a+d y+x^{2}, \quad \dot{y}=b+f y+2 x y .
$$

For these systems we calculate $Z_{6}=12 d x^{2} y$ and $Z_{7}=-12 x^{3} y\left(16 a x+f^{2} x-4 d f y\right)$. Therefore the conditions $Z_{6}=Z_{7}=0$ imply $d=0$ and $f^{2}+16 a=0$. So $a \leq 0$, and denoting $a=-\delta^{2}$ we get $f=4 \delta$. So we get the family of systems (27), i.e. in this subcase the theorem holds. Subcase $M=0$. Then for systems (24) we have $b=a=0$ and $d e \neq 0$ (otherwise we get either linear or degenerate systems). Therefore via the transformation

$$
x_{1}=x, \quad y_{1}=\frac{1}{6 d}(3 c x+6 e y+2 f), \quad t_{1}=2 e t,
$$

systems (24) will be brought to the system

$$
\dot{x}=x, \quad \dot{y}=3 y+x^{2},
$$

having the first integral $\mathcal{H}=\left(x^{2}+y\right) / x^{3}$.

Considering a quadratic system (4) according to Lemma 6 (in this case $N=Z_{1}=M=0$ ), after an additional translation (if necessary), this system can be written as

$$
\dot{x}=a+c x+d y, \quad \dot{y}=b+f y+x^{2} .
$$

For these systems we calculate $M=Z_{1}=Z_{6}=Z_{7}=0, B_{3}=6 d(f x-d y) x^{3}=0$ and hence we get $d=0$. Then $D=f^{2} x^{3}$ and $Z_{8}=8 f(f-3 c) x^{3}$. Therefore the conditions $D \neq 0$ and $Z_{8}=0$ imply $f=3 c \neq 0$. So applying the transformation

$$
x_{1}=x+\frac{a}{c}, y_{1}=-\frac{a}{c} x+c y-\frac{2 a^{2}-b c^{2}}{3 c^{2}}, t_{1}=c t,
$$

we obtain the canonical form (29).

Phase portraits. In what follows we shall determine the phase portraits of the systems belonging to the class $\mathcal{C}$ ). For this goal we shall use the results obtained in three papers: [2] to determine the configurations of the finite singularities; [16] to determine the configurations of the infinite singularities, and [22] to determine the hole phase portrait in the case that there exists a center for the system under examination. In the last case we shall denote by $V u l_{j}$ the picture with number $j$ from [22].

More precisely, from paper [2] we shall use the conditions from Table 1 given by the respective subset $S_{f i n} \subseteq \widehat{S}_{f i n}$ of invariant polynomials, where

$$
\begin{aligned}
\widehat{S}_{f i n}= & \left\{\mu, \mathbf{D}, \mathbf{T}, \mathbf{R}, \mathbf{S}, \mathbf{P}, \mathbf{U}, K, E_{1}, E_{3}, F_{1}, F_{2}, W_{1}, W_{2}, W_{3}, W_{4}, W_{7},\right. \\
& \left.V_{1}, G_{8}, G_{9}, G_{10},\left(\mathfrak{C}_{2}\right),\left(\mathfrak{C}_{5}\right),\left(\mathfrak{C}_{6}\right),\left(\mathfrak{C}_{8}\right),\left(\mathfrak{C}_{11}\right)\right\} .
\end{aligned}
$$


From paper [16] we shall use the conditions from Table 4 given by the respective subset $S_{\infty} \subseteq \widehat{S}_{\infty}$ of invariant polynomials, where

$$
\widehat{S}_{\infty}=\left\{\eta, M, \mu_{0}, \mu_{1}, \mu_{2}, \mu_{3}, \mu_{4}, L, K, K_{2}, \varkappa, \varkappa_{1}\right\} .
$$

From paper [22] we shall use the conditions provide by the Main Theorem (see [22, page $279]$ ) using the respective subset $S_{c n t} \subseteq \widehat{S}_{c n t}$ of invariant polynomials, where

$$
\widehat{S}_{c n t}=\left\{\beta, \gamma, I_{3}, I_{4}, I_{7}, I_{8}, I_{9}, I_{13}\right\} .
$$
$\mathcal{C})$.

We shall examine step by step each of the three obtained canonical forms for the class

Systems (25): $\dot{x}=x y, \dot{y}=b+e x+l x^{2}+3 / 2 y^{2}, b, l \in\{-1,0,1\}, e \geq 0$. For these systems we have $\eta=-l / 2$ and $\mu_{0}=3 l / 2$. If $\eta \neq 0$ then $\mu_{0} \neq 0$, and following [16] we conclude that the infinite singularities are determined. More precisely, since $S_{\infty}=\left\{\eta=-l / 2, \mu_{0}=\right.$ $3 l / 2, \varkappa=40 l\}$, then considering Table 4 [16] we arrive to the next result.

Remark 7 ([16]). Assume that for systems (25) the condition $\eta \neq 0$ holds. Then the behavior of the trajectories of these systems in the vicinity of infinity on the Poincaré disc corresponds to Fig. 7 (one simple node and two simple saddles) if $\eta>0$, and to Fig. 30 (one simple node) if $\eta<0$.

In what follows we shall consider three cases: $\eta>0, \eta<0$ and $\eta=0$.

Assume $\eta>0$. Then $l=-1$ and we have $U_{1}=256 b$.

Assume first $U_{1}>0$. Then $b=1$, and we get the 1 -parameter family of systems

$$
\dot{x}=x y, \quad \dot{y}=1+e x-x^{2}+3 / 2 y^{2}, \quad e \geq 0,
$$

for which

$$
S_{\text {fin }}=\left\{\mu<0, \mathbf{D}>0, K>0, W_{4}>0, W_{1}>0, V_{1}>0,\left(\mathfrak{C}_{6}\right)\right\} .
$$

So the conditions for the case 53 from [2, Table 1] are satisfied, i.e. systems above have 2 centers: $M_{1,2}\left(\left(e \mp \sqrt{e^{2}+4}\right) / 2,0\right)$. Translating the point $M_{1}$ at the origin of coordinates we obtain the systems

$$
\dot{x}=\left(e-\sqrt{e^{2}+4}\right) y / 2+x y, \quad \dot{y}=\sqrt{e^{2}+4} x-x^{2}+3 / 2 y^{2},
$$

for which $S_{c n t}=\left\{I_{13}=0, I_{9}<0, \beta>0, \gamma>4\right\}$. So according to [22] the phase portrait of these systems corresponds to $\mathcal{P}_{9}\left(V u l_{21}\right)$.

Suppose now $U_{1}<0$, i.e. $b=-1$. Then we get the systems

$$
\dot{x}=x y, \quad \dot{y}=-1+e x-x^{2}+3 / 2 y^{2}, \quad e \geq 0,
$$

for which $H_{9}=3456\left(4-e^{2}\right)$.

If $H_{9}>0$ then we have

$$
S_{f i n}=\left\{\mu<0, \mathbf{D}>0, K>0, W_{4}>0, W_{1}>0, V_{1}>0\right\},
$$

i.e. the conditions for the case 30 from [2, Table 1] are satisfied (we have two nodes). On the other hand according to Remark 7 at infinity there are one node and two saddles. Therefore taking into consideration the invariant hyperbola $-2+3 e x-6 x^{2}+3 y^{2}=0$ of systems $(31)$ passing through the finite nodes as well as the line of symmetry $y=0$, we arrive univocally to the phase portrait $\mathcal{P}_{10}$.

In the case $H_{9}<0$ (then $e>2$ ) we obtain

$$
S_{\text {fin }}=\left\{\mu<0, \mathbf{D}<0, \mathbf{R}>0, \mathbf{S}>0, K>0, W_{4}<0,\left(\mathfrak{C}_{2}\right)\right\},
$$

i.e. the conditions for the case 18 from [2, Table 1] hold. This means that systems (31) possess two nodes, one saddle and one center, which is the point $\left(\left(e+\sqrt{e^{2}-4}\right) / 2,0\right)$. Translating this point to the origin of coordinates we get the systems

$$
\dot{x}=\left(e+\sqrt{e^{2}-4}\right) y / 2+x y, \quad \dot{y}=\sqrt{e^{2}-4} x-x^{2}+3 / 2 y^{2},
$$


for which $S_{c n t}=\left\{I_{13}=0, \beta<0,4<\gamma<6, \operatorname{sign}\left(I_{3} I_{4}\right)=\operatorname{sign}\left(3 e^{2}-16\right)\right\}$. According to $[22]$ the phase portrait of these systems corresponds to $\mathcal{P}_{11}\left(V u l_{26}\right)$ if $e>4 / \sqrt{3}$, and to $\mathcal{P}_{12}$ $\left(V u l_{28}\right)$ if $2<e<4 / \sqrt{3}$. In the case $e=4 / \sqrt{3}$ following [22] for systems (32) we obtain $S_{c n t}=\left\{I_{3}=0, I_{9}-I 8<0,18 I_{7}+I_{9}-27 I_{8}>0\right\}$, and this leads to the picture $\mathcal{P}_{13}\left(V u l_{27}\right)$.

It remains to note that for systems $(31)$ we have $H_{4}=24\left(3 e^{2}-16\right)$, and hence sign $\left(3 e^{2}-\right.$ 16) $=\operatorname{sign}\left(H_{4}\right)$ and this leads to the conditions given in Table 1.

If $H_{9}=0$ we get $e=2$, and for the finite singularities of systems (31) we obtain

$$
S_{\text {fin }}=\left\{\mu<0, \mathbf{D}=0, \mathbf{T}<0, K>0, E_{1}=0, W_{2}>0\right\} .
$$

Hence the conditions for the case 73 from [2, Table 1] are fulfilled, i.e. systems (31) possess two nodes and one cusp.

Taking into consideration Remark 7 and the invariant hyperbola $-2+6 x-6 x^{2}+3 y^{2}=0$ of systems (31) (with $e=2$ ) passing through the finite nodes as well as the line of symmetry $y=0$, we arrive univocally to the phase portrait $\mathcal{P}_{14}$.

Assume finally $U_{1}=0$. Then $b=0$ and we obtain systems

$$
\dot{x}=x y, \quad \dot{y}=e x-x^{2}+3 / 2 y^{2},
$$

for which $e \in\{0,1\}$ (due to a rescaling) and $H_{4}=72 e^{2}$.

If $H_{4} \neq 0$ (i.e. $e=1$ ) then we have

$$
S_{f i n}=\left\{\mu<0, \mathbf{D}=0, \mathbf{T}=0, \mathbf{P}=0, \mathbf{R} \neq 0, E_{3}<0, G_{10}=0, W_{1}<0,\left(\mathfrak{C}_{8}\right)\right\},
$$

i.e. the conditions for the case 99 from [2, Table 1] hold. This means that system (31) (with $b=0$ and $e=1$ ) possesses the triple point $(0,0)$ (elliptic saddle) and the center $(1,0)$. Translating this point to the origin of coordinates we get the systems

$$
\dot{x}=y+x y, \quad \dot{y}=-x-x^{2}+3 / 2 y^{2},
$$

for which $S_{c n t}=\left\{I_{13}=0, \beta=0, I_{4} \neq 0,4<\gamma<6\right\}$. According to [22] the phase portrait of these systems corresponds to $\mathcal{P}_{15}\left(V u l_{24}\right)$.

Assuming $H_{4}=0$ we get $e=0$, and this leads to a homogeneous system for which we have

$$
S_{\text {fin }}=\left\{\mu<0, \mathbf{D}=0, \mathbf{T}=0, \mathbf{P}=0, \mathbf{R}=0, F_{1}=0, \eta>0, \varkappa<0\right\} .
$$

So the conditions for the case 106 from [2, Table 1] are fulfilled and hence topological phase portrait is induced by picture (e) (Fig.1)[2] and on the Poincaré disc we obtain picture $\mathcal{P}_{16}$.

Suppose now $\eta<0$, i.e. $l=1$. In this case we have $U_{1}=-256 b$.

Assume $U_{1}>0$. Then $b=-1$ and we get the 1 -parameter family of systems

$$
\dot{x}=x y, \quad \dot{y}=-1+e x+x^{2}+3 / 2 y^{2}, \quad e \geq 0,
$$

for which we calculate

$$
S_{f i n}=\left\{\mu>0, \mathbf{D}<0, \mathbf{R}>0, \mathbf{S}>0, W_{4}>0, W_{2}>0, W_{1} W_{3}>0\right\} .
$$

So the conditions for the case 4 from [2, Table 1] are verified, and hence these systems possess two saddles and two nodes.

On the other hand as $\eta<0$ by Remark 7 at infinity there exist only one simple node. Taking into consideration that all three nodes (finite and infinite) are located on the invariant straight line $x=0$ and two saddles are located on the line of symmetry $y=0$, we get univocally the phase portrait $\mathcal{P}_{17}$.

Suppose now $U_{1}<0$, i.e. $b=1$. Then we get the systems

$$
\dot{x}=x y, \quad \dot{y}=1+e x+x^{2}+3 / 2 y^{2}, \quad e \geq 0,
$$

for which $H_{9}=3456\left(e^{2}-4\right)$.

If $H_{9}>0$ then we have $e>2$ and

$$
S_{\text {fin }}=\left\{\mu>0, \mathbf{D}>0, W_{4}<0,\left(\mathfrak{C}_{5}\right)\right\} .
$$


Hence the conditions for the case 28 from [2, Table 1] are satisfied, i.e. we have one saddle and one center. Translating the center point $\left(\left(-e+\sqrt{e^{2}-4}\right) / 2,0\right)$ to the origin of coordinates we get the systems

$$
\dot{x}=\left(-e+\sqrt{e^{2}-4}\right) y / 2+x y, \quad \dot{y}=\sqrt{e^{2}-4} x-x^{2}+3 / 2 y^{2},
$$

for which $S_{c n t}=\left\{I_{13}=0, \beta \gamma>0, \gamma I_{9}>0, I_{9}(4-\gamma)<0\right\}$. According to [22] the phase portrait of these systems corresponds to $\mathcal{P}_{18}\left(V u l_{2}\right)$.

Assuming $H_{9}<0$ we obtain $0 \leq e<2$, and obviously could be determine that systems (33) have not real finite singularities, whereas at infinity according to Remark 7 there exists one simple node. Considering the invariant straight line $x=0$ we arrive to $\mathcal{P}_{19}$.

Assuming $H_{9}=0$ we get $e=2$, and for system (33) we obtain

$$
S_{\text {fin }}=\left\{\mu>0, \mathbf{D}=0, \mathbf{T}>0, E_{1}=0\right\},
$$

i.e the conditions for the case 83 from [2, Table 1] are fulfilled. Therefore this system possesses a unique finite singularity which is a cusp located on the line of symmetry $y=0$. Taking into consideration the existence of a node at infinity (see Remark 7) and the invariant straight line $x=0$, we arrive univocally to the phase portrait $\mathcal{P}_{20}$.

Suppose now $U_{1}=0$. Then $b=0$, and we obtain systems

$$
\dot{x}=x y, \quad \dot{y}=e x+x^{2}+3 / 2 y^{2},
$$

for which $e \in\{0,1\}$ (due to a rescaling) and $H_{4}=72 e^{2}$.

If $H_{4} \neq 0$ (i.e. $e=1$ ) then we have

$$
S_{\text {fin }}=\left\{\mu>0, \mathbf{D}=0, \mathbf{T}=0, \mathbf{P}=0, \mathbf{R} \neq 0, E_{3}<0, G_{10}=0\right\},
$$

i.e. the conditions for the case 88 from [2, Table 1] hold. This means that the above system (when $e=1$ ) possesses the triple point $(0,0)$ (elliptic saddle) and the saddle $(-1,0)$. By Remark 7 there is a simple node at infinity and considering the invariant straight line $x=0$ (on which the elliptic saddle is located) we get the phase portrait $\mathcal{P}_{21}$.

Assuming $H_{4}=0$ we get $e=0$ and this leads to a homogeneous system for which we have

$$
S_{\text {fin }}=\left\{\mu<0, \mathbf{D}=0, \mathbf{T}=0, \mathbf{P}=0, \mathbf{R}=0, F_{1}=0, \eta<0\right\} .
$$

So the conditions for the case 104 from [2, Table 1] are fulfilled and hence topological phase portrait is induced by picture (c) (Fig.1)[2] and on the Poincaré disc we obtain picture $\mathcal{P}_{22}$.

Assume finally $\eta=0$, i.e. $l=0$. In this case we get the systems

$$
\dot{x}=x y, \quad \dot{y}=b+e x+3 / 2 y^{2}, \quad b \in\{-1,0,1\}, e \in\{0,1\},(b, e) \neq(0,0),
$$

for which $H_{4}=72 e^{2}$ and $H_{9}=3456 b^{3} e^{2}$. So we have five concrete systems each of them being determined by the values of invariant polynomials $H_{4}$ and $H_{9}$ as follows.

If $H_{4} \neq 0$ then $e=1$, and following [16] for systems (34) we obtain $S_{\infty}=\{\eta=0, M \neq$ $\left.0, \mu_{0}=0, \mu_{1} \neq 0, \varkappa=0, L>0, K>0\right\}$. Then considering [16, Table 4] we arrive to the next result.

Remark 8 ([16]). Assume that for systems (34) the condition $H_{4} \neq 0$ holds. Then the behavior of the trajectories of these systems in the vicinity of infinity on the Poincaré disc corresponds to Fig.23 (one simple node and one topological saddle with three sectors on the the same part of the circle of Poincaré disc).

For systems (34) with $e=1$ we have $\operatorname{sign}\left(H_{9}\right)=\operatorname{sign}(b)$.

Assume first $H_{9}>0$. Then $b=1$ and for the obtained system we have

$$
S_{f i n}=\left\{\mu=0, \mathbf{D}>0, \mathbf{R} \neq 0, K>0, G_{9}=0, W_{7}<0,\left(\mathfrak{C}_{11}\right)\right\},
$$

i.e. the conditions for the case 134 from [2, Table 1] hold. This means that the system under examination possesses only one real point which is a center with the coordinates $(-1,0)$. Translating this point to the origin of coordinates we get the systems

$$
\dot{x}=-y+x y, \quad \dot{y}=x+3 / 2 y^{2},
$$


for which $S_{c n t}=\left\{I_{9}=0, I_{13}=0, I_{4} \neq 0,4<\gamma<6\right\}$. According to [22] the phase portrait of this system corresponds to $\mathcal{P}_{23}\left(V u l_{13}\right)$.

If $H_{9}<0$ then $b=-1$ and for the obtained system we have

$$
S_{\text {fin }}=\left\{\mu=0, \mathbf{D}<0, \mathbf{R} \neq 0, K>0, G_{9}=0, W_{7}>0\right\} .
$$

So the conditions for the case 117 from [2, Table 1] hold, i.e. this system possesses only three real points: the saddle $(1,0)$ and two nodes $(0, \pm \sqrt{2 / 3})$.

Taking into consideration Remark 8 and the fact that all three nodes (finite and infinite) are located on the invariant straight line $x=0$ as well as the existence of the line of symmetry $y=0$, we arrive univocally to the phase portrait $\mathcal{P}_{24}$.

Assuming $H_{9}=0$ we get $b=0$ and for system (33) we obtain

$$
S_{\text {fin }}=\left\{\mu=0, \mathbf{D}=0, \mathbf{P}=0, \mathbf{R} \neq 0, K>0, G_{10}=0\right\},
$$

i.e the conditions for the case 147 from [2, Table 1] hold. Therefore the system (34) with $e=1$ and $b=0$ possesses a unique (triple) finite singularity which is an elliptic saddle located at the intersection of the line of symmetry $y=0$ with the invariant line $x=0$.

Taking into consideration Remark 8 we obtain univocally the phase portrait $\mathcal{P}_{25}$.

Finally suppose $H_{4}=0$, i.e. $e=0$, and then $b \in\{-1,1\}$. In this case for systems (34) we have $H_{6}=256 b y^{6}$, and hence $\operatorname{sign}\left(H_{6}\right)=\operatorname{sign}(b)$.

If $H_{6}>0$ then $b=1$ and we obviously detect that system (34) with $e=0$ and $b=1$ has no real finite singularity. On the other hand following [16] for this system we obtain

$$
S_{\infty}=\left\{\eta=0, M \neq 0, \mu_{0}=0, \mu_{1}=0, \mu_{2}>0, \varkappa=0, \varkappa_{1}=0, L>0, K_{2}<0\right\},
$$

and according to [16, Table 4] for the infinite singular points we get Fig.8 (i.e. one node and one two-separatrices saddle). Considering the invariant line $x=0$ and the line of symmetry $y=0$ we arrive to the phase portrait $\mathcal{P}_{26}$.

In the case $H_{6}<0$ we get $b=-1$, and for system (34) with $e=0$ and $b=-1$ we obtain

$$
S_{\text {fin }}=\left\{\mu=0, \mathbf{R}=0, \mathbf{P} \neq 0, \mathbf{U}>0, K>0, G_{8}=0, F_{2}<0\right\},
$$

i.e the conditions for the case 164 from [2, Table 1] are fulfilled and we obtain two nodes. For the infinite singular points we have

$$
S_{\infty}=\left\{\eta=0, M \neq 0, \mu_{0}=0, \mu_{1}=0, \mu_{2}<0, \varkappa=0, \varkappa_{1}=0, L>0, K>0\right\}
$$

and according to [16, Table 4] we get Fig.27 (i.e. one node and multiple point with six saddle sectors). As all three nodes (finite and infinite) are located on the invariant straight line $x=0$, and considering the line of symmetry $y=0$ we obtain the phase portrait $\mathcal{P}_{27}$.

Systems (27): $\dot{x}=x^{2}-\delta^{2}, \dot{y}=b+4 \delta y+2 x y, \delta, b \in\{0,1\},(\delta, b) \neq(0,0)$. As we can observe this family consists of three distinct systems, for which we calculate $H_{6}=-32768 \delta^{2} x^{6}$ and $B_{3}=12 b x^{4}$.

If $H_{6} \neq 0$ then $\delta=1$, and following [16] for systems (27) we obtain $S_{\infty}=\{\eta=0, M \neq$ $\left.0, \mu_{0}=0, \mu_{1}=0, \mu_{2}>0, L<0, \varkappa=0, \varkappa_{1}=0\right\}$. Then considering [16, Table 4] we conclude that the behavior of the trajectories of these systems in the vicinity of infinity on the Poincaré disc corresponds to Fig. 17, i.e. there are one simple saddle and one singularity of multiplicity 4 (having two elliptic sectors, each one of the different parts of infinity).

On the other hand for the finite singularities, following [2], we obtain

$$
S_{\text {fin }}=\left\{\mu=0, \mathbf{R}=0, \mathbf{P} \neq 0, \mathbf{U}>0, K>0, G_{8}=0, F_{2}>0\right\},
$$

i.e the conditions for the case 150 from [2, Table 1] hold. Therefore systems (27) with $\delta=1$ possesses two finite singularities: one node and one saddle.

If $B_{3}=0$ (i.e. $b=0$ ), then the finite singular points are located on the invariant straight line $y=0$ which are at the same time the separatrices of the infinite saddles. Moreover, this invariant straight line is also a line of symmetry of the respective vector field of the system (27) with $\delta=1$ and $b=0$. Considering the existence of two invariant straight lines $x= \pm 1$ passing through the finite singularities we get univocally the phase portrait $\mathcal{P}_{29}$. 
In the case $B_{3} \neq 0$ we have $b=1$, and clearly in this case the there does not exist a separatrix connection between finite and infinite saddles because the flow along the segment $\{(x,-1 / 2), x \in(-\infty,-1)\}$ goes always upwards. Taking into account the invariant straight lines $x= \pm 1$ and the locations of all the singularities (finite and infinite) we obtain the phase portrait $\mathcal{P}_{30}$.

Assume now $H_{6}=0$, i.e. $\delta=0$. Then $b=1$ and we get the concrete system

$$
\dot{x}=x^{2}, \dot{y}=1+2 x y,
$$

which evidently has not finite singularities. To determine the configuration of infinite singularities following [16] we obtain

$$
S_{\infty}=\left\{\eta=0, M \neq 0, \mu_{0}=0, \mu_{1}=0, \mu_{2}=0, \mu_{3}=0, \mu_{4}>0, L<0, \varkappa=0, \varkappa_{1}=0\right\} .
$$

Then considering [16, Table 4] we conclude that the behavior of the trajectories of this system in the vicinity of infinity on the Poincaré disc corresponds again to Fig.17, but besides the simple saddle there is one singularity of multiplicity 6 (having two elliptic sectors, each one of the different parts of infinity).

Taking into consideration the double invariant straight line $x=0$ and the facts that the change $(x, y, t) \mapsto(-x-y-t)$ keeps this system (i.e. the origin of coordinate is a center of symmetry of its phase portrait) and that the flow along $x$-axis goes upward we arrive to picture $\mathcal{P}_{31}$.

III. System (29): $\dot{x}=x, \dot{y}=3 y+x^{2}$. Evidently the unique simple singular point $(0,0)$ of this system is a node.

To examine infinite singularities, following [16], we obtain

$$
S_{\infty}=\left\{M=0, \mu_{0}=0, \mu_{1}=0, \mu_{2}=0, \mu_{3} K_{1}>0, K_{3}<0\right\}
$$

i.e. according to $[16$, Table 4] the behavior of the trajectories of this system at infinity corresponds to Fig. 38 (a unique singular point of multiplicity six, having two hyperbolic and two parabolic sectors which alternate as it is indicated in Fig. 38 [16]).

As the finite node is located on the invariant straight line $x=0$ and this line is also the line of symmetry for the system under consideration we get the phase portrait $\mathcal{P}_{28}$.

\section{ACKNOWLEDGMENTS}

The first two authors have been supported by the grants MCD/FEDER number MTM200803437 and CIRIT 2001SGR 00173. The third author has been partially supported by CRDF-MRDA CERIM-1006-06.

\section{REFERENCES}

[1] J. C. ARtés, J. Llibre AND N. I. Vulpe, Quadratic systems with a rational first integral of degree 2 : a complete classification in the coefficient space $\mathbb{R}^{12}$, Rendiconti del Circolo Matematico di Palermo 56 (2007), 417-444.

[2] J. C. Artés, J. Llibre And N. I. Vulpe, Singular points of quadratic systems: A complete classification in the coefficient space $\mathbb{R}^{12}$, International J. of Bifurcation and Chaos 18 (2008), 313-362.

[3] J.C. Artés, J.Llibre AND N. Vulpe, Quadratic systems with a polynomial first integral: a complete classification in the coefficient space $\mathbb{R}^{12}$, J. Differential Equations 246 (2009), 3535-3558.

[4] V. Baltag, Algebraic equations with invariant coefficients in qualitative study of the polynomial homogeneous differential systems, Bull. of Acad. of Sci. of Moldova. Mathematics, 2003, no. 2 , 31-46

[5] V.A. Baltag And N.I. Vulpe, Total multiplicity of all finite critical points of the polynomial differential system, Planar nonlinear dynamical systems (Delft, 1995), Differential Equations \& Dynam. Systems 5 (1997), 455-471.

[6] L. Cairó AND J. Llibre, Phase portraits of quadratic polynomial vector fields having a rational first integral of degree 2, Nonlinear Anal., Theory, Methods and Appl. 67 (2007), 327- 348.

[7] IU. CALIN, On rational bases of $G L(2, \mathbb{R})$-comitants of planar polynomial systems of differential equations, Bull. of Acad. of Sci. of Moldova. Mathematics, 2003, no. 2, 69-86

[8] J. Chavarriga, B. García, J. Llibre, J.S. PÉrez del Río, J.A. Rodríguez Polynomial first integrals of quadratic vector fields J. Differential Equations 230 (2006), 393-421.

[9] B. García, J. Llibre And J.S. PÉRez Del Río, Phase portraits of the quadratic vector fields with a polynomial first integral, Rendiconti del Circolo Matematico di Palermo A 55 (2006), 420-440. 
[10] J. Llibre And R. D. S. Oliveira, Phase portraits of quadratic polynomial vector fields having a rational first integral of degree 3, Nonlinear Anal., Theory, Methods and Appl. 70 (2009), 3549-3560.

[11] J. Llibre And R. D. S. OliveirA, Correction to "Phase portraits of quadratic polynomial vector fields having a rational first integral of degree 3", Nonlinear Anal., Theory, Methods and Appl., 2009

[12] P.J. Olver, Classical Invariant Theory, London Mathematical Society student texts 44, Cambridge University Press, 1999.

[13] H. Poincaré, Sur l'intégration des équations différentielles du premier ordre et du premier degré I and II, Rendiconti del circolo matematico di Palermo 5 (1891), 161-191; 11 (1897), 193-239.

[14] M.N. Popa, Applications of algebraic methods to differential systems, Romania, Piteshty Univers., The Flower Power Edit., 2004 (Romanian).

[15] D. Schlomiuk And N. Vulpe, Planar quadratic differential systems with invariant straight lines of at least five total multiplicity, Qualitative Theory of Dynamical Systems, 5 (2004), 135-194.

[16] D. Schlomiuk And N. Vulpe, Geometry of quadratic differential systems in the neighborhood of infinity, J. Differential Equations 215 (2005), 357-400.

[17] D. Schlomiuk AND N. Vulpe, Integrals and phase portraits of planar quadratic differential systems with invariant lines of at least five total multiplicity, Rocky Mountain J. Math. 38 (2008), 2015-2076.

[18] D. Schlomiuk And N. Vulpe, Planar quadratic differential systems with invariant straight lines of total multiplicity four, Nonlinear Anal., Theory, Methods and Appl. 68 (2008), 681-715.

[19] D. Schlomiuk And N. Vulpe, The full study of planar quadratic differential systems possessing a line of singularities at infinity, J. Dynam. Differential Equations 20 (2008), 737-775.

[20] D. Schlomiuk And N. Vulpe, Integrals and phase portraits of planar quadratic systems with invariant lines of total multiplicity four, Bul. Acad. Ştiinţe Repub. Mold. Mat. 56 (2008), 27-83.

[21] K.S. SibIRSKII, Introduction to the algebraic theory of invariants of differential equations, translated from the Russian, Nonlinear Science: Theory and Applications, Manchester University Press, Manchester, 1988.

[22] N.I. VulPE, Affine-invariant conditions for the topological discrimination of quadratic systems with a center, Differential Equations 19 (1983), 273-280.

[23] N.I. Vulpe, Polynomial bases çof comitants of differential systems and their applications in qualitative theory (Russian), "Shtiintsa", Kishinev, 1986.

[24] J. W. REYN, A bibliography of the qualitative theory of quadratic systems of differential equations in the plane, Delf University of Technology, http://ta.twi.tudelf.nl/DV/Staff/ J.W.Reyn.html, 1997

[25] Ye Yanqian And others, Theory of Limit Cycles, Transl. Math. Monographs 66, Amer. Math. Soc., Providence, 1984.

1 Departament de Matemàtiques, Universitat Autònoma de Barcelona, 08193 Bellaterra, BARCElona, Spain

E-mail address: artes@mat.uab.cat, jllibre@mat.uab.cat

2 Institute of Mathematics and Computer Science, Academy of Science of Moldova, 5 Academiei Str, ChişinĂU, MD-2028, Moldova

E-mail address: nvulpe@mail.md 